\title{
Flow Separation Side Loads Excitation of Rocket Nozzle FEM
}

\author{
Kurt B. Smalley NASA MSFC \\ Dr. Andrew Brown NASA MSFC \\ Joseph Ruf NASA MSFC \\ Dr. John Gilbert UAH
}

Introduction

Modern rocket nozzles are designed to operate over a wide range of altitudes, and are also built with large aspect ratios to enable high efficiencies. Nozzles designed to operate over specific regions of a trajectory are being replaced in modern launch vehicles by those that are designed to operate from earth to orbit. This is happening in parallel with modern manufacturing and wall cooling techniques allowing for larger aspect ratio nozzles to be produced.

Such nozzles, though operating over a large range of altitudes and ambient pressures, are typically designed for one specific altitude. Above that altitude the nozzle flow is 'underexpanded' and below that altitude, the nozzle flow is 'overexpanded'. In both conditions the nozzle produces less than the maximum possible thrust at that altitude. Usually the nozzle design altitude is well above sea level, leaving the nozzle flow in an overexpanded state for its start up as well as for its ground testing where, if it is a reusable nozzle such as the Space Shuttle Main Engine (SSME), the nozzle will operate for the majority of its life. Overexpansion in a rocket nozzle presents the critical, and sometimes design driving, problem of flow separation induced side loads.

If ambient pressure on the outside of the nozzle is at a greater pressure than that of the nozzle flow, it is possible for the ambient pressure to force its way upstream along the walls causing the flow to separate from the nozzle wall and a recirculation zone to appear. Recirculation zones cause adverse pressure gradients within the supersonic flow, thus creating shock waves extending from the nozzle wall.

If the nozzle flow separates, a pressure differential between the inner and outer nozzle surfaces is created. If the separation is asymmetric a resulting transverse or 'side load' is created. Nozzle side loads can cause damage to the nozzle itself as well as to engine support structures such as the gimbal joint, actuators or thrust adaptor.

Flow separation induced side loads have caused failures during ground testing of multiple rocket engines. In the SSME, the three coolant feed lines that run down the outside of the nozzle from the forward to aft manifolds had weld ruptures that were determined to be partially due to unforeseen high loading from nozzle side loads during hot fire [6]. In the J-2 engine, gimbal bolts failed in tension from large nozzle side loads during ground testing [8]. Nozzle flow separation occurs within a nozzle when the ratio of nozzle wall pressure to ambient pressure falls below a critical pressure ratio. This ratio is passed through during start-up and shut down transients. For engines that are able to throttle, such as the SSME, the engine can operate steady state, at or below the critical pressure ratio for a significant amount of time.

In an overexpanded nozzle asymmetric flow separation appears and disappears in a random manner. Because of this, the characteristics of the side load are usually described in a statistical manner. Also, when the compliance of the nozzle structure allows for large deformations of the nozzle to interfere with the internal flow field, an aeroelastic coupling phenomenon can take 
effect. This phenomenon can have a potentially damaging amplification effect on nozzle deformations.

In order to design a nozzle to withstand side loads, engineers usually err on the side of conservatism to ensure the design will withstand the maximum amplitude the nozzle might incur. Thus, the lack of an accurate model of the rocket nozzle side load will force a designer to use over conservative empirical models of the phenomenon which tend to over-design the rocket nozzle. The over-design can result in a heavy weight and cost penalty on the nozzle.

With a goal of creating accurate predictive models of the rocket nozzle flow separation side load, NASA Marshall Space Flight Center (MSFC) personnel have carried out a multi-year experimental and analytical investigation into the phenomenon. One of the results of these studies was the formulation of a fluid dynamic analysis methodology that generates a set of internal pressure contours for a nozzle undergoing steady state two nodal-diameter aeroelastic flow separation for a single instance in time. This methodology was applied to predict the internal pressure field of a subscale 27" long nozzle that was cold flow tested in the MSFC Nozzle Test Facility.

This paper will discuss the implementation of this coarse but reasonable representation of the pressure field spatial and temporal characteristics onto a structural dynamic finite element model of the nozzle in a time transient analysis. The resulting model structural response is then compared to test to validate the methodology used to create the pressure field. This comparison revealed that the analysis deformations were significantly higher than those measured during testing. On the other hand the analytically predicted shape and frequency of the nozzle response were both accurate. If the pressure contour is reformulated according to a measurement based amplification factor, it is hoped that an accurate yet simple prediction of the nozzle response to the aeroelastic flow separation side load can be obtained.

\section{Background}

To increase their understanding of nozzle side loads, engineers at MSFC began an investigation in 2000 into the phenomenon through a task entitled "Characterization and

- Accurate Modeling of Rocket Engine Nozzle Side Loads" [1, 2, 3], led by A. Brown. The stated objective of this study was to develop a methodology to accurately predict the character and magnitude of nozzle side loads. The study included further hot-fire testing of the MC- 1 engine, cold flow testing of subscale nozzles, CFD analyses of both hot-fire and cold flow nozzle testing, and finite element (f.e.) analysis of the MC-1 engine and cold flow tested nozzles. A follow on task included an effort to formulate a simplified methodology for modeling a side load during a two nodal diameter fluid/structure interaction for a single moment in time.

Two cold flow nozzle test article were manufactured. The first was a thick walled truncated ideal nozzle that is stiff enough to not deflect an appreciable amount due to side loads. This nozzle was used to baseline the pure fluid physics of the experiment. The second nozzle, seen in Figure 1, had the same internal contour but had a much thinner wall. This nozzle was used to characterize any fluid structure interaction. Both nozzles were 26.7" long and 12.4" diameter at their exit planes with 30 to 1 area ratios. 


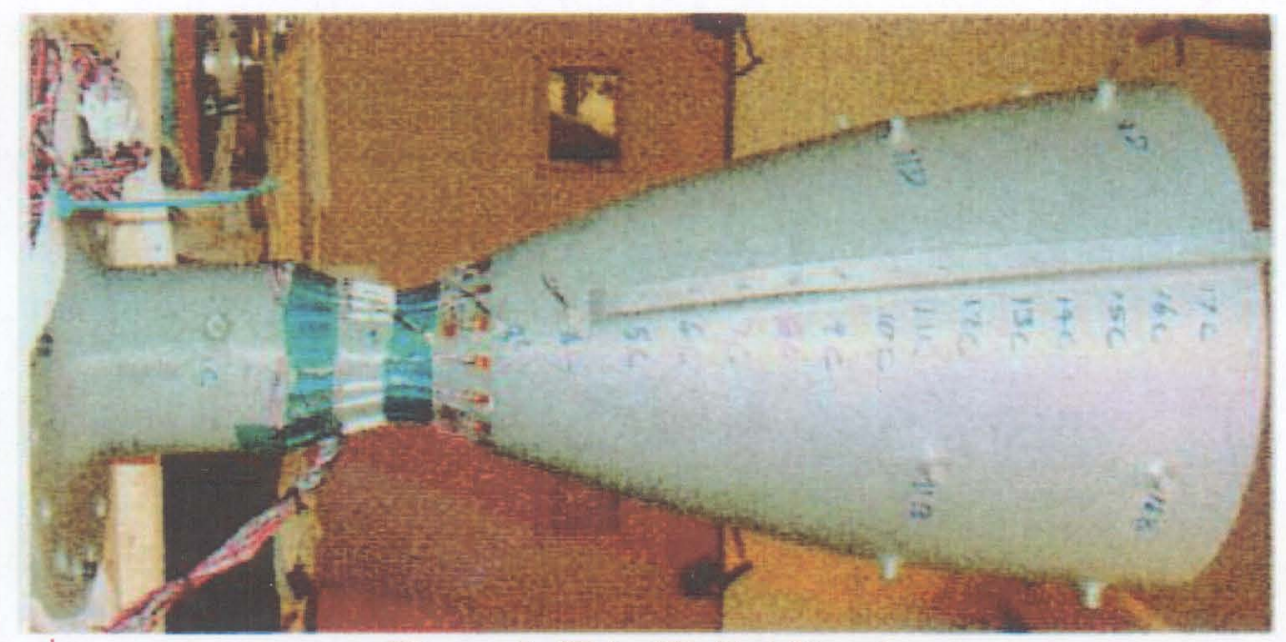

Figure 1. Thin Walled Nozzle

A modal test was performed on both the NTF nozzle support structure and the thin walled nozzle itself. Details and results of this testing can be found in $[4,5]$. The results of the modal tests were used to determine the natural frequency of the thin walled nozzle second nodal diameter mode as well as to ground a finite element model of the nozzle which was used to aid in an investigation of the nozzle/support structure coupling problem. The finite element model of the nozzle and test facility back up structure (see Figure 3) was created by MSFC engineering in MSC.PATRAN. The high fidelity of the model was used to ensure good strain predictions from the experimental strain gage locations and was verified through comparison with the modal test. For time transient response analyses, the facility/nozzle interface was constrained as a cantilever boundary condition.

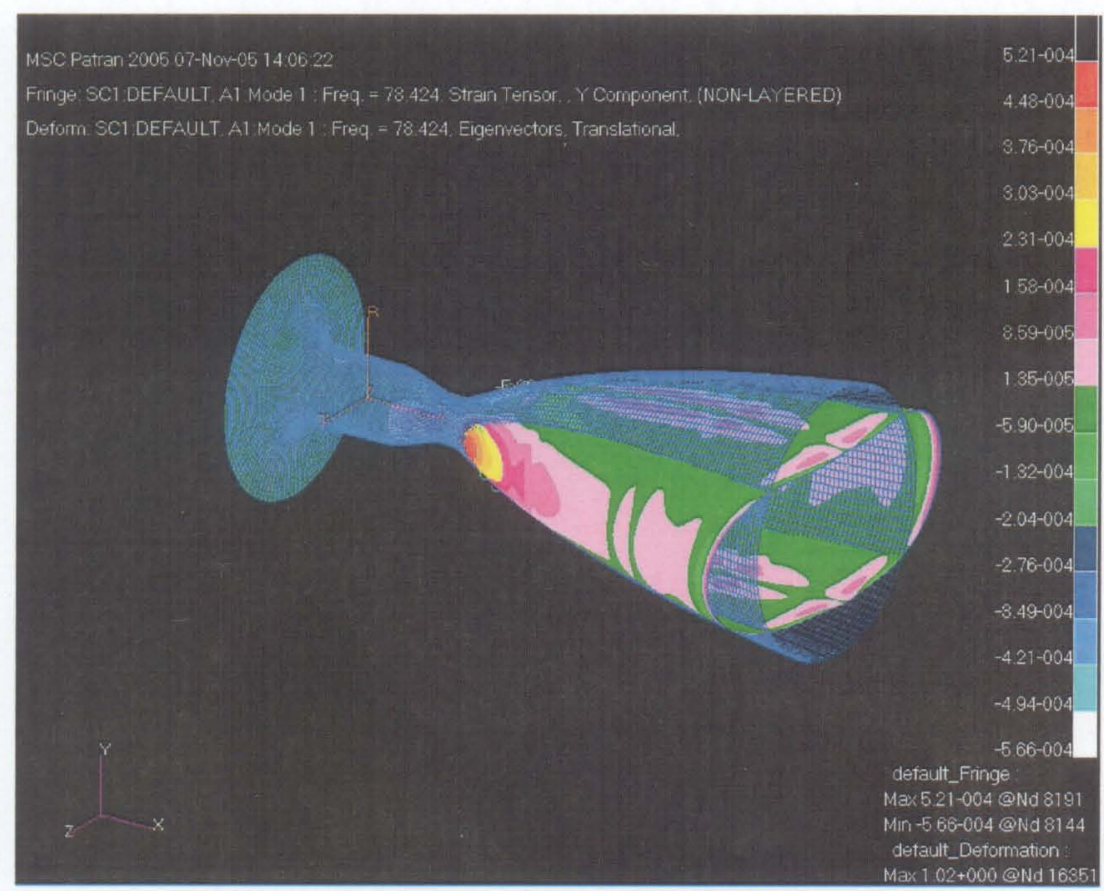

Figure 3. FEM of Thin Walled Nozzle 
The cold flow tests were conducted at MSFC in the Nozzle Test Facility (NTF) as seen in Figure 2. Compressed dry air was the nozzle working fluid. The nozzles were mounted in the test cabin. The pressure in the cabin was varied via an ejector system.

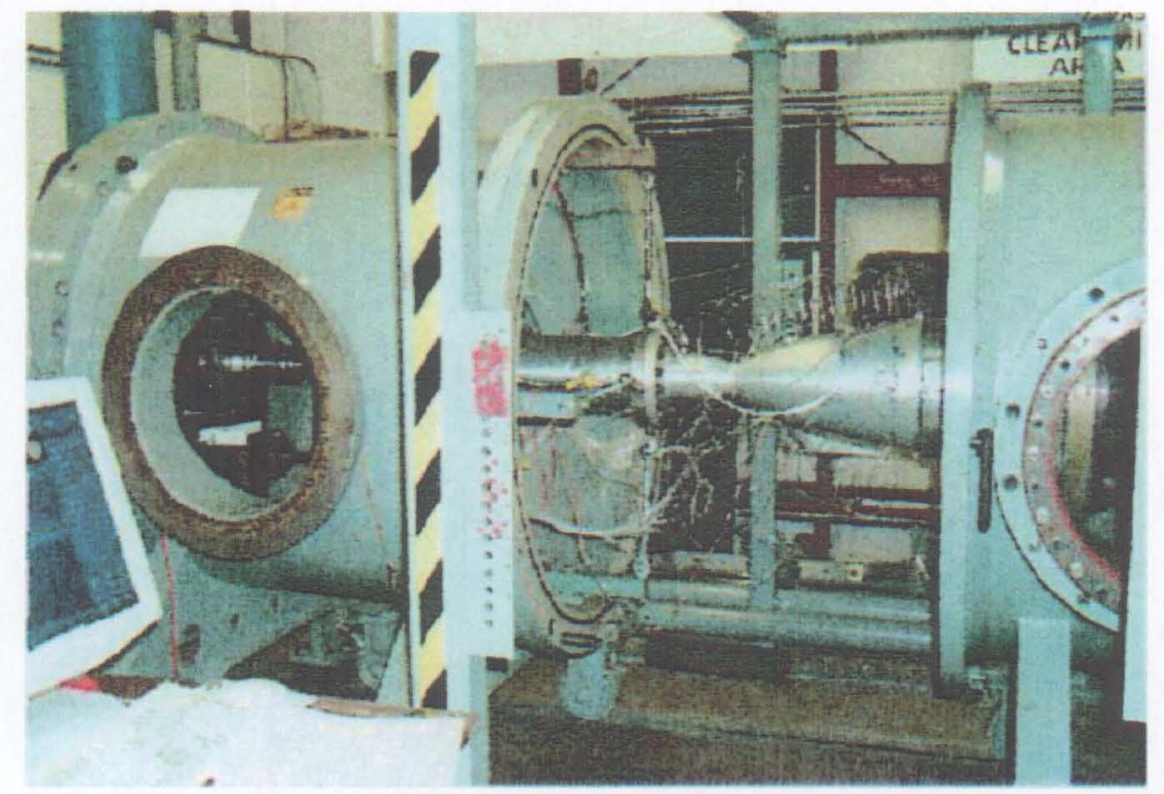

Figure 2. Thick Walled Nozzle in NTF

Sixteen circumferential strain gages were mounted onto the flexible walled nozzle just downstream of the nozzle throat, every 22.5 degrees. Three axial strain gages were mounted just upstream of that. Steady and fluctuating pressures were mounted onto the nozzle in two axial and two radial planed ribs on each nozzle. Also, four accelerometers were mounted on the thick walled nozzle, two on the thin.

Originally, load cells were to be mounted radialy at the nozzle facility interface. Unfortunately, due to a nozzle/facility feedback problem during operation, these load cells had to be removed to prevent damage to them and the facility. Both nozzles were tested over a range of mass flow rates, inlet total pressures, test cabin pressures and nozzle pressure ratios. Further detail on this testing can be found in $[9,10]$.

As expected, the thin walled nozzle showed a much larger response on the strain gage data as well as the fluctuating pressure data. A survey of the thin walled nozzle data showed that for every high strain region during the testing, the nozzle second nodal diameter was the dominant frequency in the strain gage data. Further analyses of the strain gage data showed that this frequency did indeed coincide with a two nodal diameter shape. The fluctuating pressure data also showed a high nozzle two nodal diameter frequency response.

A discrepancy with the data was revealed, though, when a comparison of the phase between the strain gage data and the pressure data revealed that they were in phase. This is ninety degrees off of what would be expected for a resonant case. This problem led to the investigators inability to prove with certainty if this response truly was a result of the fluid/structure interaction. 


\section{Methodology}

Based on the cold flow results, MSFC personnel formulated a fluid dynamic analysis methodology that generates a set of internal pressure contours for a nozzle undergoing steady state two nodal-diameter aeroelastic flow separation for a single instance in time. This methodology would then be assessed by applying this pressure field in a structural dynamic transient response analysis and comparing the results with experimental data.

The first step in this methodology is to create deformed nozzle contours representing the two nodal diameter shape of the nozzle at different clocking positions. The highest strain gage responses were taken from the cold flow testing performed at MSFC NTF and comparing them to mass normalized strains from a finite element modal analysis of the nozzle. The strain gage responses were taken during run 37 3_sep at 24.6 seconds.

This comparison yielded a strain scale factor. The mass normalized deflection of the modal analysis was then multiplied by this scale factor in order to obtain representative deformations of a nozzle running axially from the combustion chamber to the nozzle exit of the test article.

A series of nozzle wall contours was extracted from the finite element model of the nozzle deformed in the 2ND mode shape. Axial wall contours were extracted at constant circumferential angles of $0,22.5,45,67.5$ and 90 degrees. These contours were used to develop a representative load distribution for the deformed nozzle with separated flow. Symmetry of the 2ND deflection shape allowed these locations to be reflected to represent the remainder of the nozzle.

The second step of the methodology was to generate nozzle internal flow fields for each of the deformed profiles. Towards this end, a two-dimensional kinetics (TDK) methodology was used. The TDK analyses assumed the nozzles were full flowing, i.e., no separation. Each contour was analyzed independently as an axisymmetric nozzle. Nozzle total conditions were derived from the test data. Wall pressure distributions were extracted from the TDK solutions. These can be seen in Figure 4.

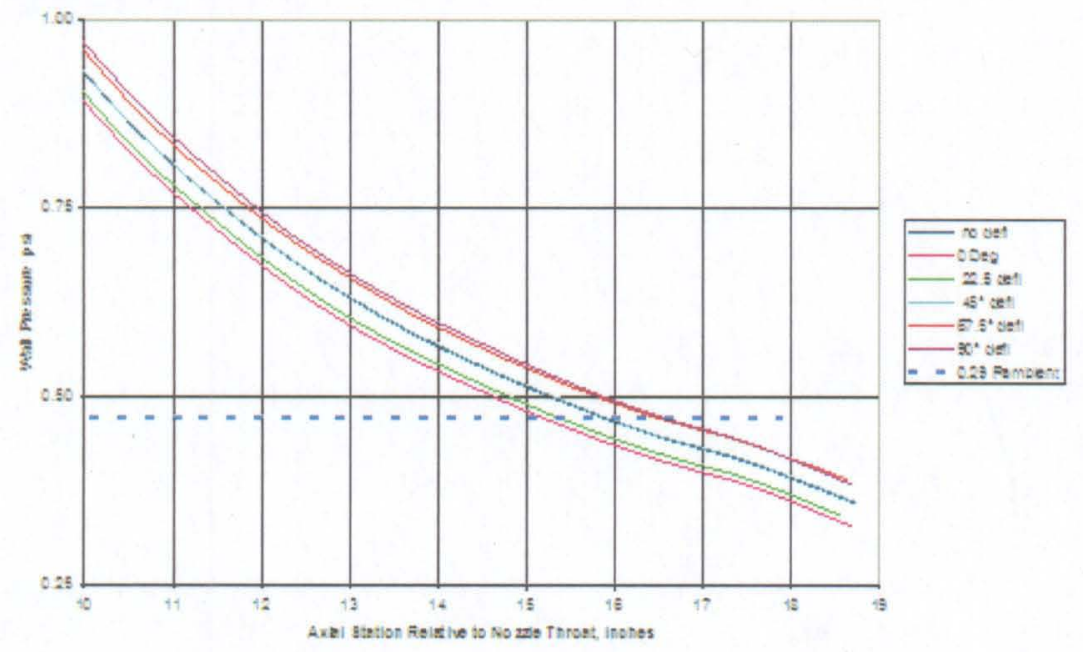

Figure 4. TDK Extracted Wall Pressure Distribution

The separation location was then estimated. The nozzle flow separates at a location where the wall pressure is between 0.28 to 0.38 of ambient pressure. For this analysis, a value of 0.29 was used to choose a separation location. A line representing 0.29 of the cabin pressure for the 
test point in run 37_3 sep at 24.6 seconds can be seen in Figure 5. This is a time in which the test article was excited in the $2 \mathrm{ND}$ mode. The location at which each of the contour pressure distributions crossed the $0.29 \mathrm{P}_{\text {ambient }}$ curve was set as its separation location.

The pressure downstream of each contour's separation location was assumed to rise to 0.98 of ambient pressure. The resulting pressure distributions are shown in Figure 5.

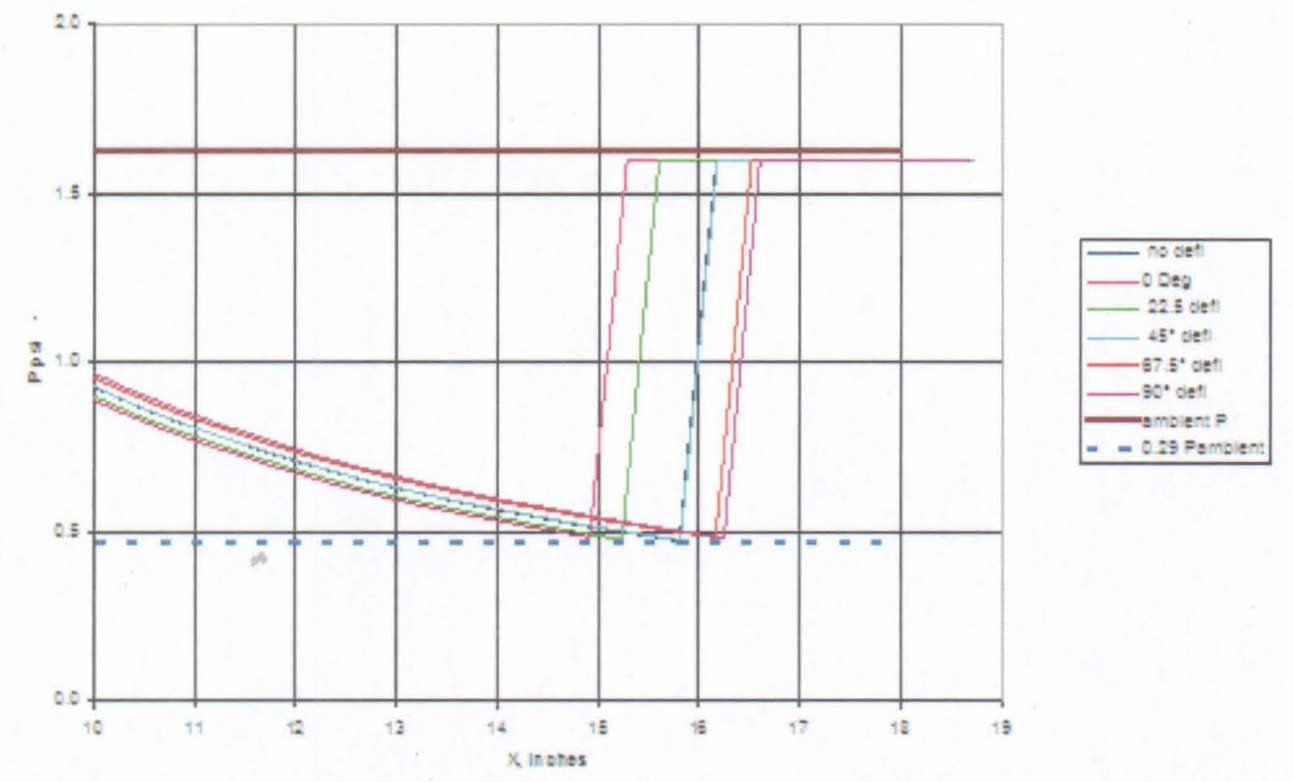

Figure 5. Nozzle Wall Pressure Distributions with Assumed Separation

This approach assumed 0.29 for the separation location. Values of 0.28 to 0.38 could have also been used. The magnitude of the forces would have been different, but the relative magnitudes would have been the same.

Dynamic effects were ignored in the flow analysis and thus a fixed wall condition was assumed. This is a good assumption because the aerodynamics of the nozzle change much quicker than the shape of the nozzle. This means the nozzle aerodynamics will quickly adjust to a new wall shape. This assumption allows for the methodology to produce a model of steady state flow separation which is undergoing a fluid/structure interaction with the nozzle for a single moment in time. Furthermore, this assumption also allows for the methodology to be validated via a structural time transient analysis of the nozzle, since the aerodynamics would be able to keep up with any changes in the nozzle geometry.

The method of validation for the internal pressure contours for a nozzle was to convert the pressure contours into force time history forcing functions. These forcing functions are then applied to the nozzle finite element model in a time transient structural response analysis from which the nozzle strain results are compared back to the original test data. The pressure contours consist of absolute pressure vs. axial location down the length of the nozzle for 6 different circumferential clockings.

In order to apply the original pressure contours in a time transient analysis, the contours first had to be manipulated in order to give them the correct spatial discretization. To spatially discretize the pressure contours down to a manageable number of application points, six planes moving axially down the nozzle were chosen. Each plane was roughly 3.18 " apart. Around each 
plane, 16 nodes were then chosen as points of force excitation for the transient analysis, making a total of 96 excitation locations symmetrically placed around the nozzle. An illustration of these points can be seen in Figure 7.

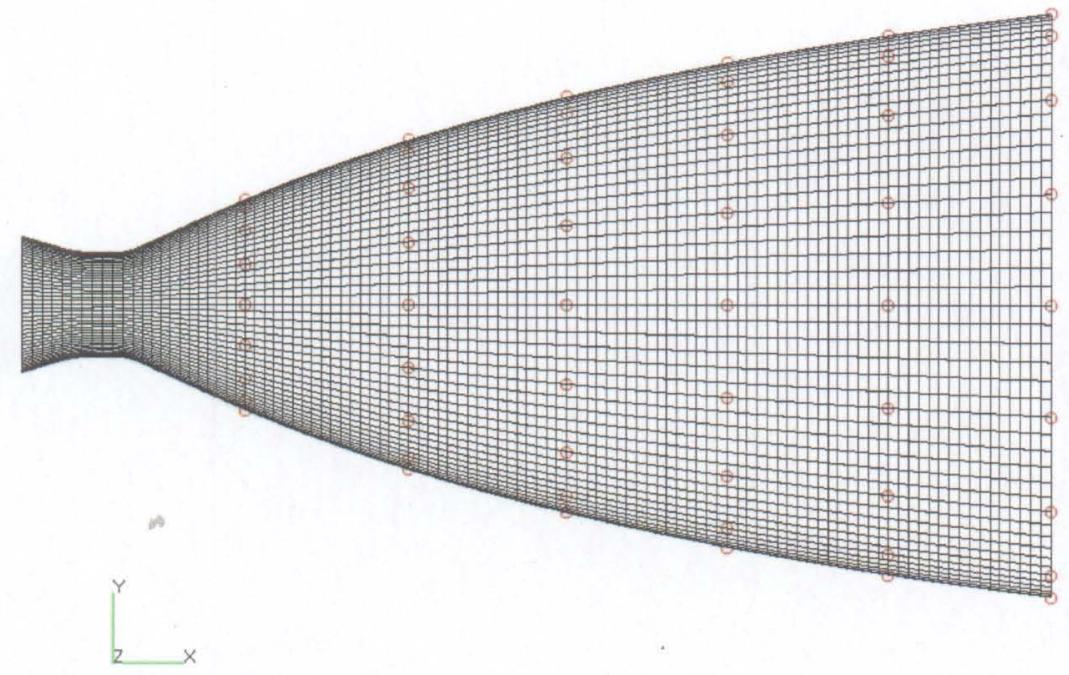

Figure 7. Nozzle Model Excitation Points

Next, the ambient pressure within the test diffuser was subtracted out of the contours. In the case of this experiment, this pressure was $1.7 \mathrm{psi}$. This was done to produce the gage pressure acting across the nozzle wall. It should be noted that the subtraction of the ambient pressure from the pressure field negated the pressure field within the plane at the end of the nozzle, making it zero. This fact allowed for the subtraction of the last plane of excitation points from the analysis. Finally, the pressure contours were interpolated to span a full 360 degrees, creating a two nodal diameter shape.

After the spatial interpolation, the time transient aspect of the forcing function was applied. This was achieved by calculating the mean pressure around each axial plane in the pressure field.

Each value in the pressure field matrix was then given a time varying component by fluctuating it around its respective mean value. This was done by simply multiplying each point by

$$
P_{f}(j, i)=m(j)+(P(j)-m(j)) * \sin (2 * \pi * 78.4 * t(i)),
$$

where $\mathrm{P}_{f}(\mathrm{i}, \mathrm{j})$ is the fluctuating wall pressure in both circumferential clocking position (j) and time (i), $P(j)$ is the static wall pressure, $m(j)$ is the mean pressure around a circumferential plane and $t(i)$ is a time array. The frequency of oscillation was chosen as the analytical two nodal diameter mode. The excitation points of interest were then extracted out of the full pressure field matrix. 
The pressure field next had to be transformed into a force field in order to be applied to the nodes within the model. This transformation was done by calculating an effective area around each node and multiplying each pressure forcing function by its effective area.

It should be noted that one additional manipulation was necessary to manipulate the pressure fields into realistic time variant representations. At the second-to-last plane of excitation down the nozzle, a sinusoid could not be used to truly represent the time variant nature of the force field.

As the separation plane passes each individual point in this plane, the pressure jumps from the ambient to the pressure upstream of the separation bubble. This transition happens very rapidly. The pressure at any given point will then remain constant until the separation plane passes again. For this reason, a square wave was chosen to represent the time variant aspect of the forces in this plane. The circumferential loading distribution for a single time point in the analysis along the 6 chosen planes can be seen in Figure 8 .

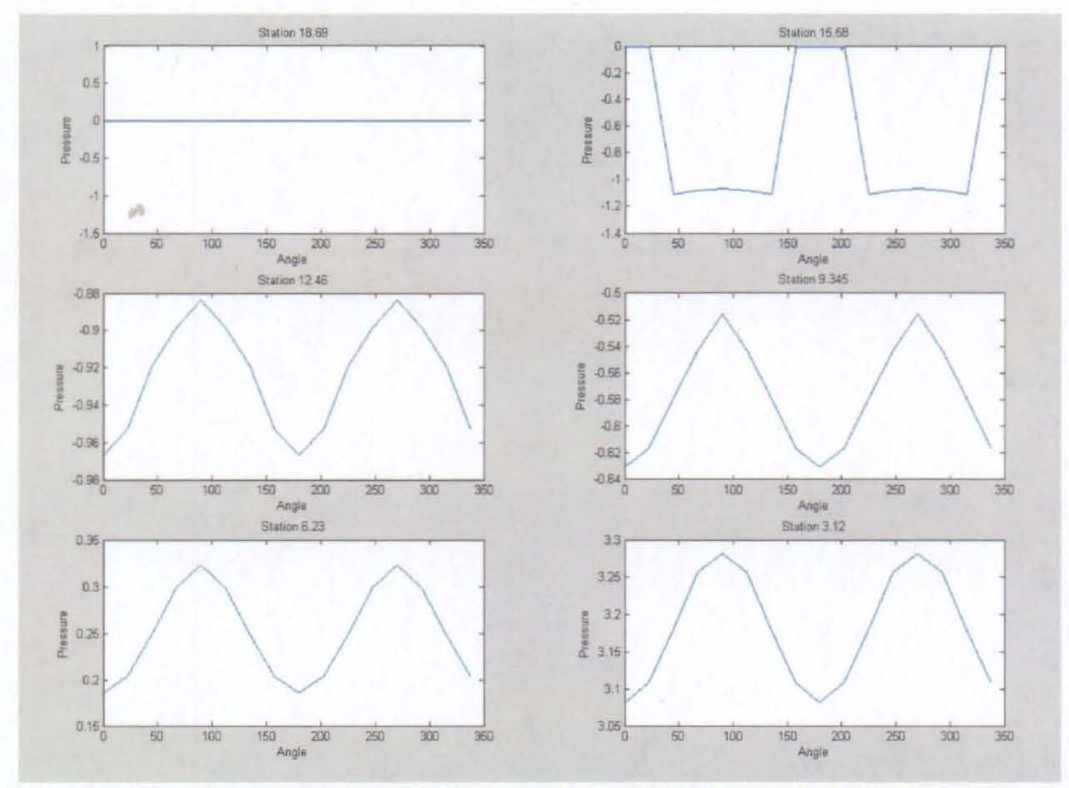

Figure 8. Circumferential Loading Distribution For a Single

The forces were applied to their respective node points in a non-initial condition transient analysis in the commercial code NASTRAN. The time duration for the analysis was chosen to ensure that the analysis would obtain a steady state solution, from which the maximum strains would be used to compare back to the test. The time step of the analysis was chosen to be 0.0001 seconds with 256 time steps. This number of time steps allowed for a Fast Fourier Transform to be performed on results, enabling Power Spectral Density operations during post processing.

The analytical magnitudes of the analysis are dependant on the damping value used in the analysis. For that reason, two damping values were used in two separate analyses in hopes to bound the analytical results. The first damping value used was the pure modal damping of 0.22 percent critical damping obtained through the impact modal test. This value, in a resonant condition, is used as a minimum damping that should exist in the structure during operation. 
The second damping value used as the upper bound value was obtained by a quality factor calculation performed on both the strain gage and accelerometer data. The quality factor can be calculated as follows:

$$
Q=\frac{F_{n}}{F_{2}-F_{1}},
$$

where $F_{n}$ is the resonant frequency of interest and $F_{1}$ and $F_{2}$ are the frequencies of the $1 / 2$ power points of that resonant peak. For a power spectral density curve, the $F_{1}$ and $F_{2}$ values lie at $1 / \sqrt{ } 2$ the $F_{n}$ amplitude. The quality factor can then be related to the critical damping ratio as follows:

$$
\zeta=\frac{1}{2 Q} \text {. }
$$

Though this calculation gives only a rough estimation on the true damping value of the nozzle during operation, it was thought to be accurate enough in this application to act as an upper bound. The quality calculation revealed that the experimental damping factor was roughly 0.8 percent critical damping. The operational modal damping value was found to be slightly larger than the impact test modal damping.

\section{Results}

From the NASTRAN forced response transient analysis, strain results were obtained in the nozzle circumferential and axial directions at the nozzle cold flow strain gage locations. The results were in the form of strain time history and were later converted into strain spectral density plots. The analytical circumferential strain time histories and PSD can be seen in Figures 9 and 10. The comparable test circumferential stain gage data can be seen in Figures 11 and 12 respectively. To view the experimental data, the ASRI commercial software PC Signal was used.
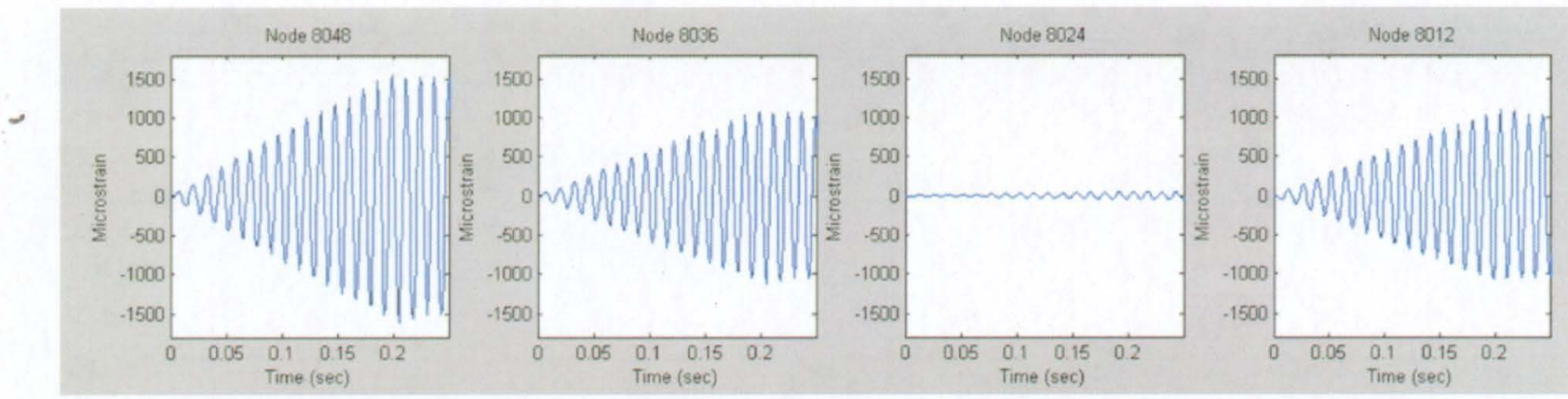

Figure 9. Analytical Circumferential Strain Time History Results 

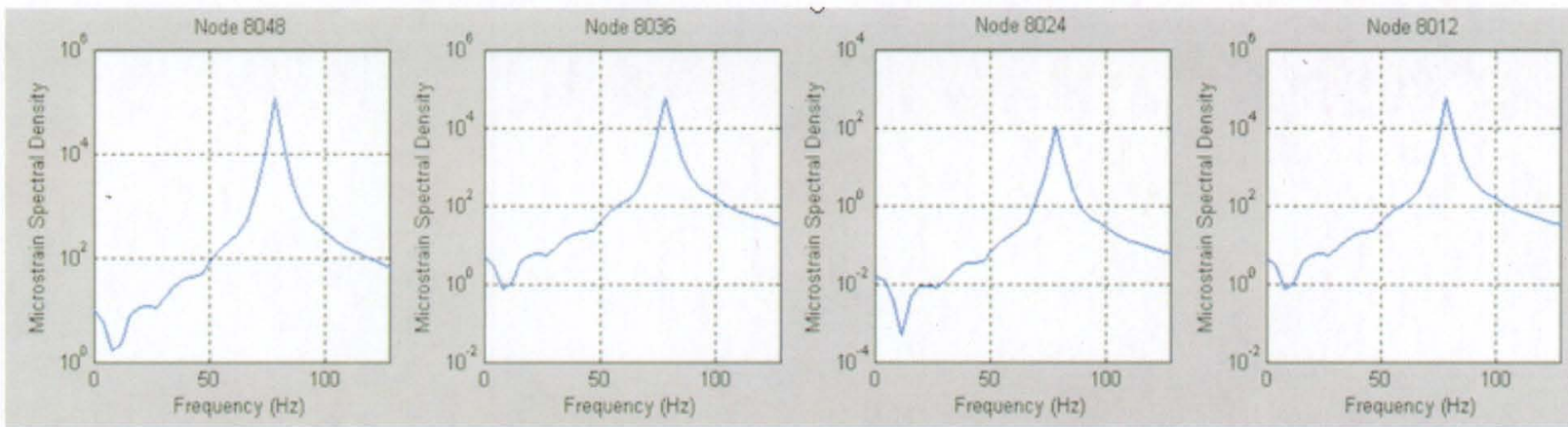

Figure 10. Analytical Circumferential Strain PSD Results
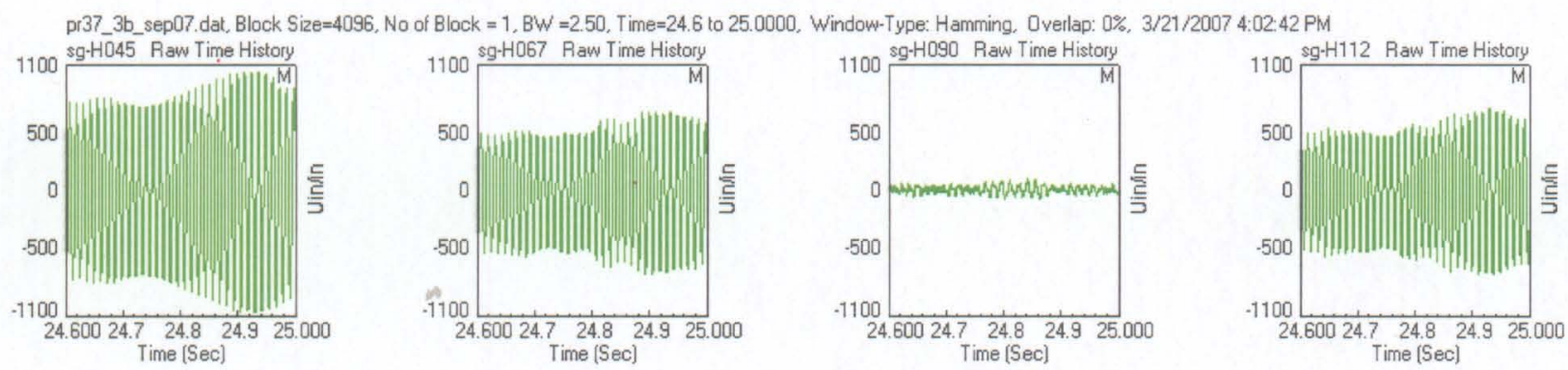

Figure 11. Experimental Circumferential Strain Time History Results

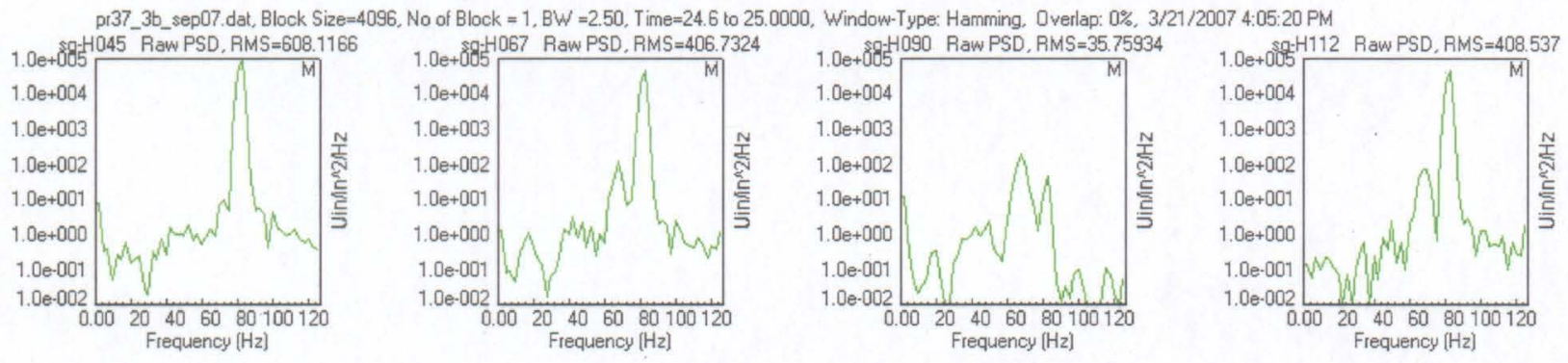

Figure 12. Experimental Circumferential Strain PSD Results

It can be seen in both the strain spectral densities and time histories, the frequency content of the test vs. the analysis compare well. This was to be expected since the forcing functions were crafted to have the same frequency as the analytical two nodal diameter mode.

It can also be seen in the comparison between the analytical strain values and the test values that both have two nodal diameter shapes. This can be seen through the clocking of the strain gages. Both sets of data show a definite trend of increasing from a noise floor to the highest amplitude and back again over 180 degrees. Even the relative amplitudes from gage to gage are similar.

The greatest difference between the analytical and experimental results can be seen in the strain time history circumferential amplitudes. The analytical amplitudes are almost $100 \%$ greater than that of the test. It should be noted again that the analytical magnitudes are dependant on the damping value used in the analysis. In this particular run, the pure modal damping of 0.22 percent critical damping was used. 
The results of the analysis using the quality factor calculated modal damping showed a decrease in the maximum expected strain at the strain gage locations of approximately $13 \%$ from the original analysis; still being $60 \%$ over the test data.

One possible explanation for the over-prediction of the analytical results could be as follows. The methodology was derived in order to take into account the possible fluid/structure interaction of the system at a single moment in time through an iterative process between structural analysis and fluid analysis. The basis of the structural analysis is the creation of nozzle deformed contours representing the shape of the excited nozzle during operation.

These contours, as discussed previously, are based on operational strain gage measurements of the nozzle dynamic response to the fluid forcing function. The nozzle should deflect a given amount due to an applied pressure in a static application. The nozzle will then deflect that amount times a dynamic amplification factor in a dynamic resonance condition due to that same applied pressure.

The dynamic amplification factor is a function of the damping of the dynamic system. The nozzle pressure contours from the fluid analysis were formulated using an assumed deflection equal to $10 \%$ of the measured dynamic deflection of the nozzle. Since the TDK analysis assumed rigid walls, this is the equivalent to assuming the $10 \%$ deflection was due to the static pressure. This would allow for the $90 \%$ deflection unaccounted for in the pressure field to be taken into account by the dynamic amplification of the nozzle in resonance with the fluid.

Any difference in this assumed deflection would have direct impact to the final analytic strain amplitudes. If indeed the amplification factor takes into account a greater portion of the resonance deformed shape than the $90 \%$ allocated, the difference between the two would be in fact double booked by both the amplification factor in the transient analysis and the static deflection in the original nozzle deformed contour. The choice of $10 \%$ deflection chosen was developed from engineering judgment.

The appropriate way to test this theory would be to replace the use of the $10 \%$ deflection shape to derive the internal pressure contours with one derived from a dynamic quality factor calculated from the experimental response data. For this particular case, a quality factor of 62.5 could be used, yielding a 1.6\% deflection shape.

This theory was tested by repeating the transient dynamic analysis using an analytic damping factor of 0.05 , which would equate to an amplification factor of $90 \%$. The results of this analysis revealed strains that were $50 \%$ of the test results, a substantial over-correction. At this point, therefore, the hypothesis remained unproven.

For this analysis, only circumferential results were used in the comparisons. An attempt was originally made to perform an axial comparison as well. This attempt yielded poor results. An attempt was also made to compare axial to circumferential strain ratios between test and analysis to show a similar strain field. This attempt was also shown to have poor results.

A second possible explanation for the over-prediction of the analytical results could be the chosen location of nozzle flow separation. Nozzle flow separation occurs at a location where the wall pressure is between 0.28 to 0.38 of ambient pressure. For this analysis, a value of 0.29 was used to choose a separation location. A higher value would have decreased the difference between the forces applied to the nozzle ninety degrees apart, thus decreasing the strain in the nozzle.

Only three axial measurements were taken during the experiment, two of which were at node points, leaving only one measurement to use for such comparisons. To further the difficulty in 
performing the comparison, the finite element analysis showed that for the two nodal diameter shape, the axial strain gages were in a high strain gradient region (Figure 13).

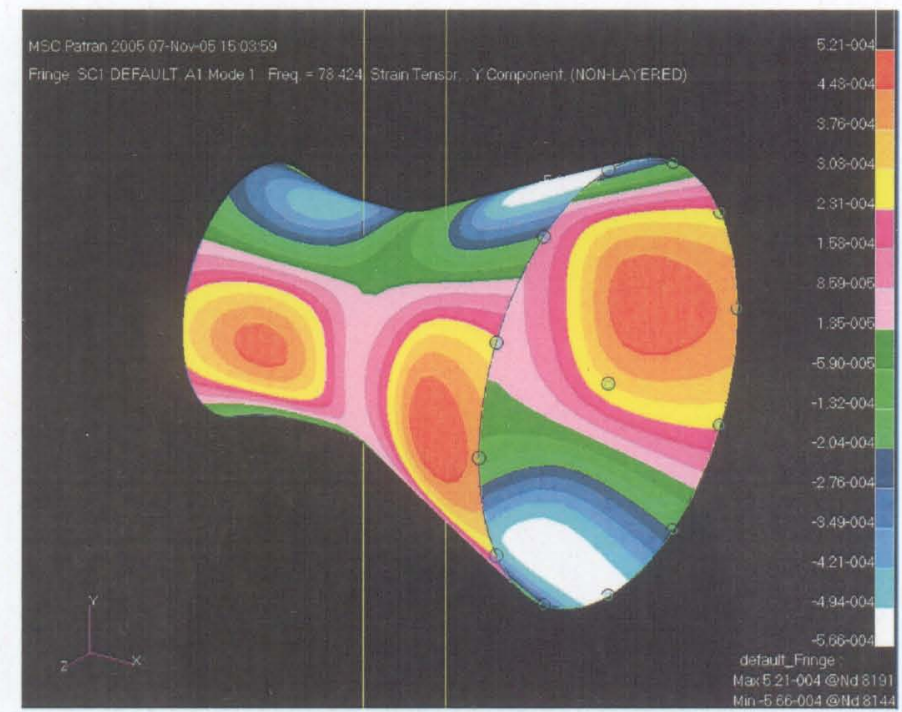

Figure 13. Experimental Strain Gage Plane Location

Any small error in the location of the strain gage location, therefore, would result in a large error in the magnitude of the strain measured. For these reasons, these attempts were abandoned.

An interesting and accidental result of this study was that any slight difference between the analytical two nodal diameter frequency and the forcing function frequency would result in a beating effect of the transient strain response. This result was uncovered when a first iteration forcing function accidentally used the experimental two nodal diameter frequency instead of the analytic.

Looking at the analytic strain fields of this analysis showed a modal strain field that was most certainly not a two nodal diameter shape. Once the forcing function frequency was aligned with - the analytical two nodal diameter frequency, the beating disappeared. Thus, this result shows that only a forcing function at the same frequency as nozzle two nodal diameter response could create a true nodal diameter shape. Since the experiment showed a locking in of this frequency and mode shape over an appreciable duration, it can be argued that the fluid forcing function had to be at the two nodal diameter frequency and shape, and thus organized to be that way by the structure.

\section{Conclusion}

Researchers at NASA Marshall Space Flight Center undertook a comprehensive experimental and analytical study of the rocket nozzle flow separation induced side load phenomenon. One of the goals of this study was to derive a simplified analytical model of the internal pressure field of a rocket nozzle during an aeroelastic side load phenomenon in a single instance in time. The simplified analytical model discussed in this paper allows for the use of strain gage data, TDK analysis and a finite element analysis to predict a pressure field, and thus a nozzle side load forcing function, from engine hot fire or cold flow testing.

An attempt was made to validate this simplified analytical model. The method of validation was to apply the simplified analytical model derived pressure field to a time transient analysis of a finite 
element model of a nozzle. Initial results indicate that the derived forcing function over-predicted the strain response of the nozzle. Possible sources of error are in the formulation of the supplied forcing functions in the assumed experimental dynamic amplification factor of the nozzle/fluid system when scaling from experimental data, or in the chosen location of nozzle flow separation.

It is hoped that future work on this project includes the application of the suggestions from this paper as well as an additional validation of the updated methodology. Beyond this work, the application of a model pressure field derived from a 3D CFD analysis is being looked into. The advantage of a 3D analysis would be to include the 3D flow effects of the phenomenon. This would allow the flow to move circumferentially around the nozzle as well as axially.

Once a derived pressure field is validated successfully, the proven methodology used to derive that pressure field can then be applied to analyses in support of the development of future rocket engines. This capability would be useful for full scale engine analyses where the mounting of strain gages during hot fire testing is tractable, while the mounting of fluctuating pressure transducers is not. The simplified analytical model would also allow for, with the use of subscale nozzle test pressure data scaled up to full scale nozzles, predictions of nozzle side load forcing functions to be made without the testing of the full scale engine. Thus structural dynamic analyses could be performed on a full scale engine as a design cycle analytical assessment before that engine is ever manufactured or hot fired.

[1] Brown, Dr. Andrew M., "Characterization And Accurate Modeling of Rocket Engine Nozzle Side Loads," NASA Marshall Space Flight Center, Huntsville, AL, Center Director's Discretionary Fund Proposal, 2000.

[2] Brown, Dr. Andrew, "Characterization And Accurate Modeling of Rocket Engine Nozzle Side Loads," NASA Marshall Space Flight Center, Huntsville, AL, FY01Center Director's Discretionary Fund Annual Report, February 2002.

[3] Brown, Dr. Andrew M., Ruf, Joe, Reed, Darren, D’Agostino, Mark and Keanini, Dr. Russell, "Characterization Of Side Load Phenomena Using Measurement of Fluid/Structure Interaction," AIAA 2002-3999, 2002.

[4] Driskill, Tim, "Sub-Scale, Thin Wall Nozzle Test Facility Modal Surveys," NASA Marshal Space Flight Center, Huntsville, AL, Modal Test Report NTF-DEV-01-057, 2001.

[5] Driskill, Tim, "Sub-Scale, Nozzle and Nozzle Test Facility Modal Survey and Checkout Procedure As Run," NASA Marshal Space Flight Center, Huntsville, AL, Modal Test Procedure NTF-DEV-01-057, 2001.

[6] Larson, Edward W., Ratekin, Gary H. and O'Conner, George M., "Structural Response To The SSME Fuel Feedline To Unsteady Shock Oscillations," Rockwell International, Rocketdyne Division, Canoga Park, CA.

[7] Ruf, Joseph and McDaniels, Dave, "Nozzle Side Load Technology - Second Year Proposal for $2^{\text {nd }}$ Year of a Two Year CDDF 03-09," NASA, Marshall Space Flight Center, Huntsville, AL, CDDF Report, 2004. 
[8] Rocketdyne Engineering, "Side Load Test And Analysis," North American Rockwell, Rocketdyne Engineering, Canoga Park, CA, Presentation, 1974.

[9] Snellgrove, Lauren, "Characterization And Accurate Modeling of Rocket Engine Side Loads Nozzle Air Flow Test," NASA Marshal Space Flight Center, Huntsville, AL, Post Test Report, Test Number P2223, November, 2001.

[10] Snellgrove, Lauren, "Characterization And Accurate Modeling of Rocket Engine Side Loads Nozzle Air Flow Test Requirements Document (TRD)," NASA Marshal Space Flight Center, Huntsville, AL, Internal Memo TD63 (01-0013), May, 2001. 


\section{Flow Separation Side Loads Excitation of Rocket Nozzle FEM}

Kurt B. Smalley, Andrew Brown, Ph.D., and Joseph Ruf NASA/MSFC, Huntsville AL, 35812

John Gilbert, Ph.D.

University of Alabama in Huntsville, Huntsville, AL 35899 


\section{Overview}

- Significance of Work

- Explanation of rocket nozzle side loads

- Brief history of work done at MSFC

- Simplified model of nozzle internal pressure field during aeroelastic separation

- Validation attempt and results

- Possible sources of error

- Conclusions 


\section{Motivation and Purpose of this Study}

- Rocket nozzle side loads have caused failures on the test stand for the:

- Space Shuttle Main Engines "steer horn"

- Saturn V J-2 Engine gimbal bolts

- Japanese LE-7A actuators and regenerative cooling tubes.

- Inaccurate and over-conservative modeling techniques cause over-design of nozzle, payload penalties.

- Create a more accurate model of phenomena

- reduce side load related failures

- redue weight and payload penalties on launch vehicles.

- Create simplified 2D fluid dynamics model and determine if it can accurately predict experimental observations. 


\section{Side Loads in Rocket Nozzles}

- During start-up, shut-down, or sea-level testing of high-altitude engine, ambient pressure higher than much of internal nozzle wall pressures.

- Boundary layer separation of low-pressure internal fluid flow from inner wall of nozzle

- In-rushing ambient pressure at uneven axial locations causes large transverse shock load

- Caused failures of both nozzle actuating systems and sections of the nozzle itself.

- Existing side load calculation method

- Assumes separation at two different axial stations, integrates the resultant $\triangle \mathrm{P} * \mathrm{dA}$ loads.

- Method calibrated to maximum and minimum possible separation locations to be intentionally conservative.

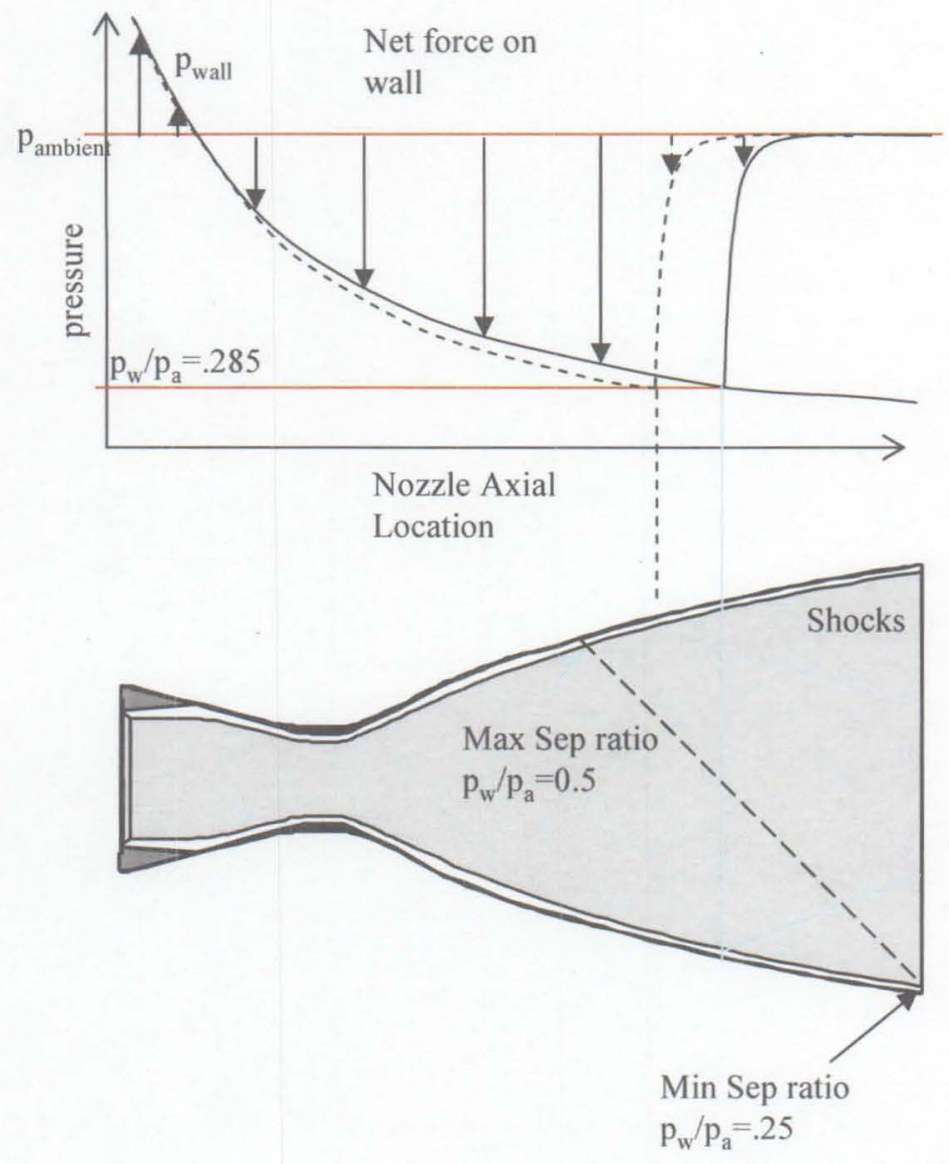




\section{MSFC Side Loads Research Program during FASTRAC, 1998}

- During design, knew FASTRAC engine would operate in overexpanded condition during ground test.

- Test/analysis program initiated during FASTRAC/MC-1 project to obtain physics-based, predictable value.

- Strain-gauge measurements taken on nozzle during hot-fire test Nov. 1998

- Flow separation clearly identified at Steady-State Operation.
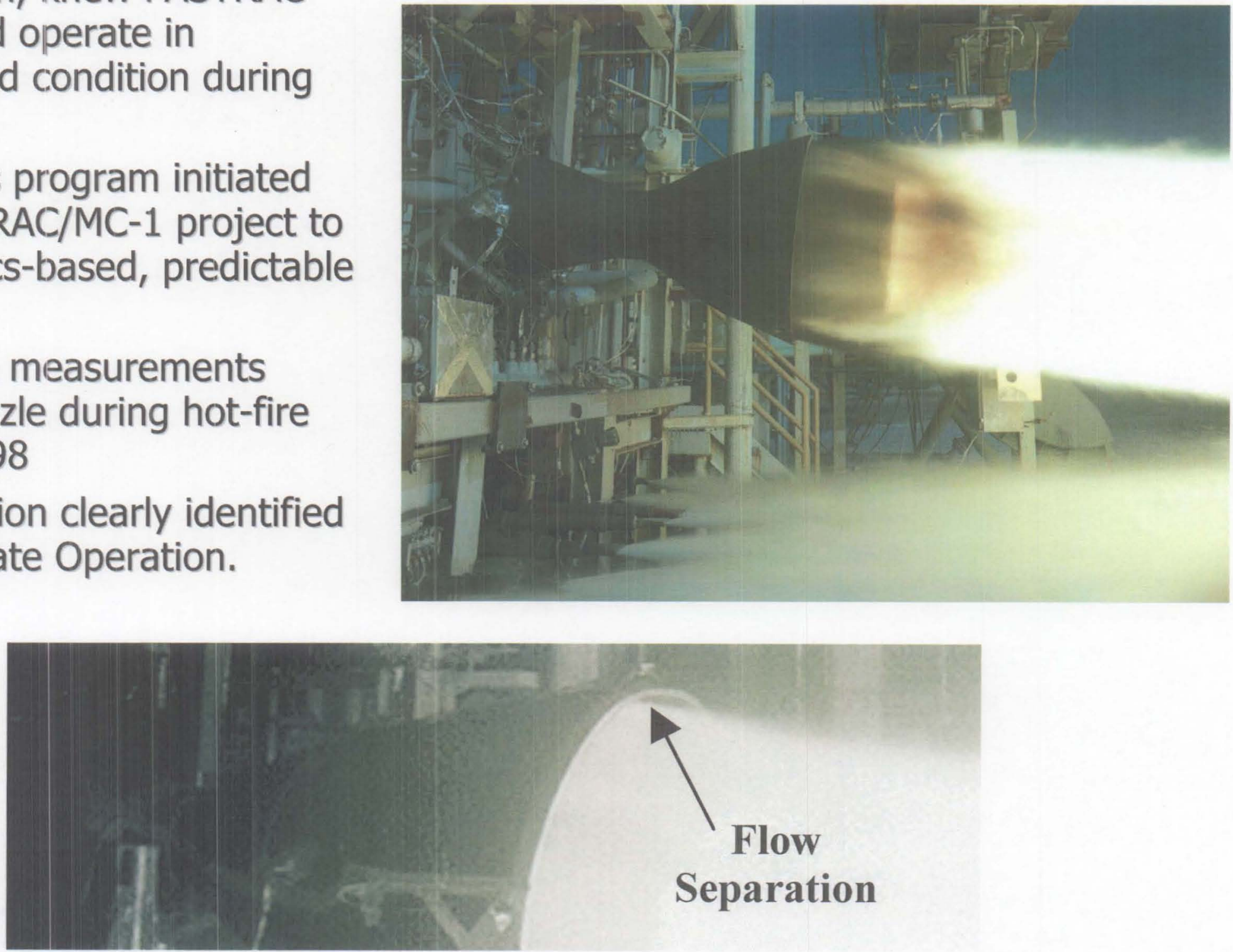
NASA
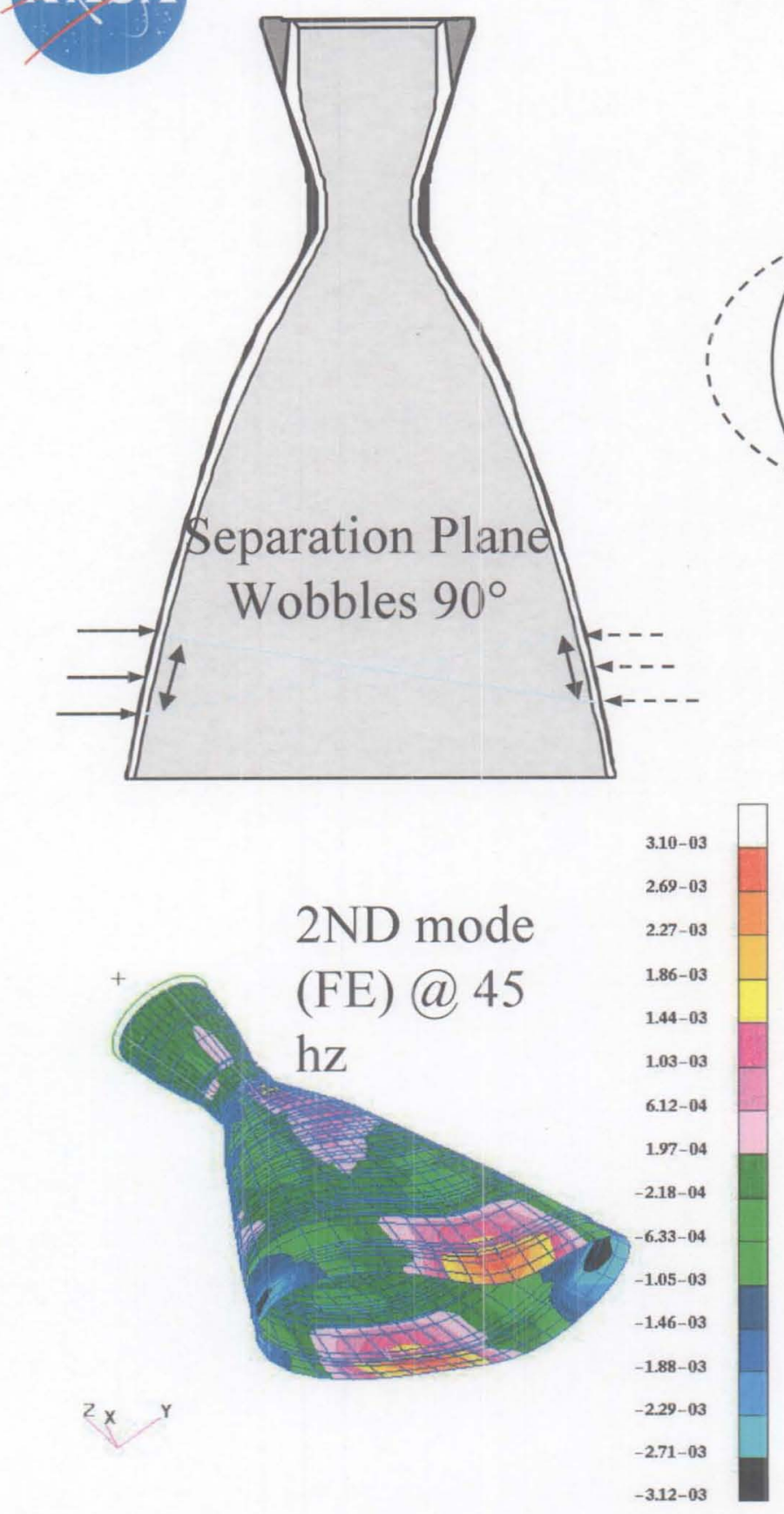

Steady-State Separation.

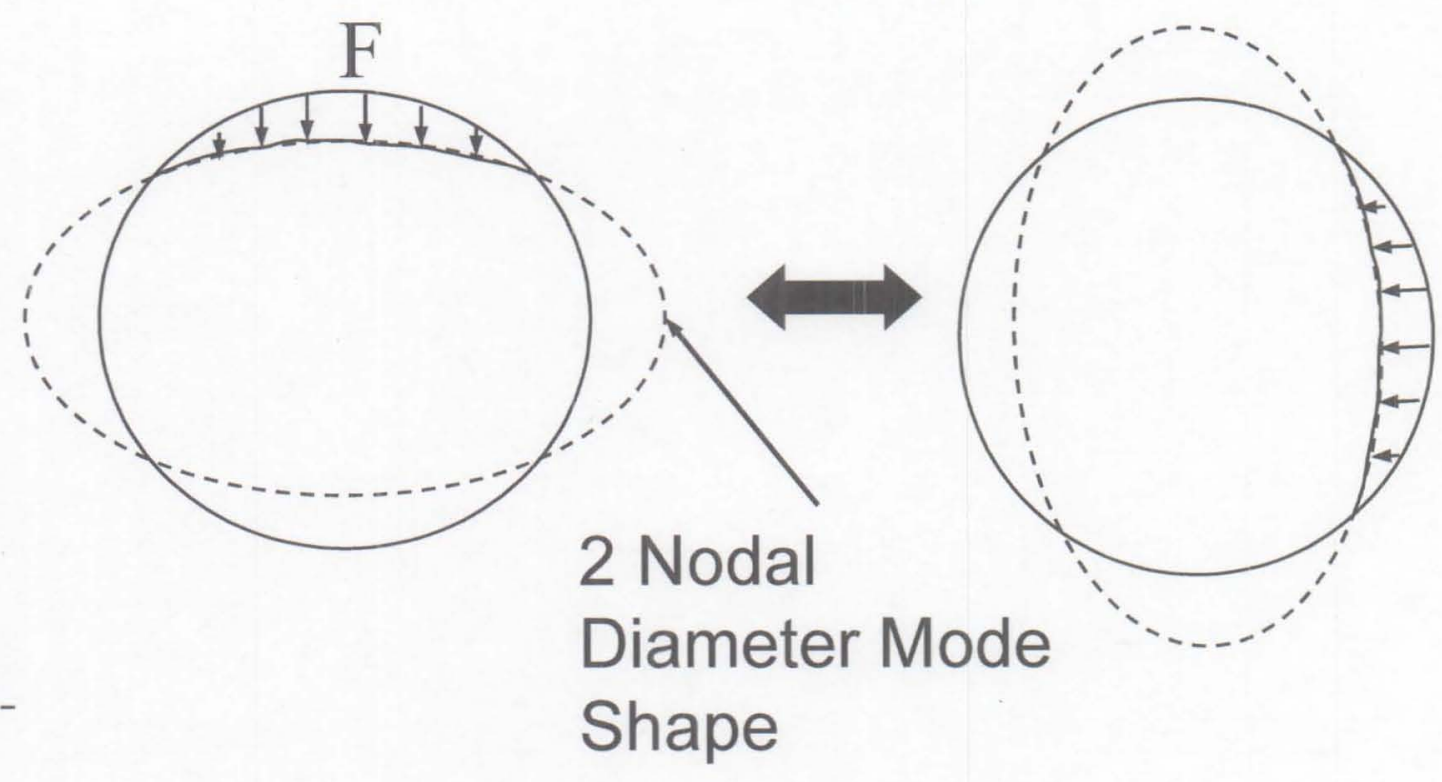

FASTRAC Hot-Fire test Strain time histories at 16 circumferential locations

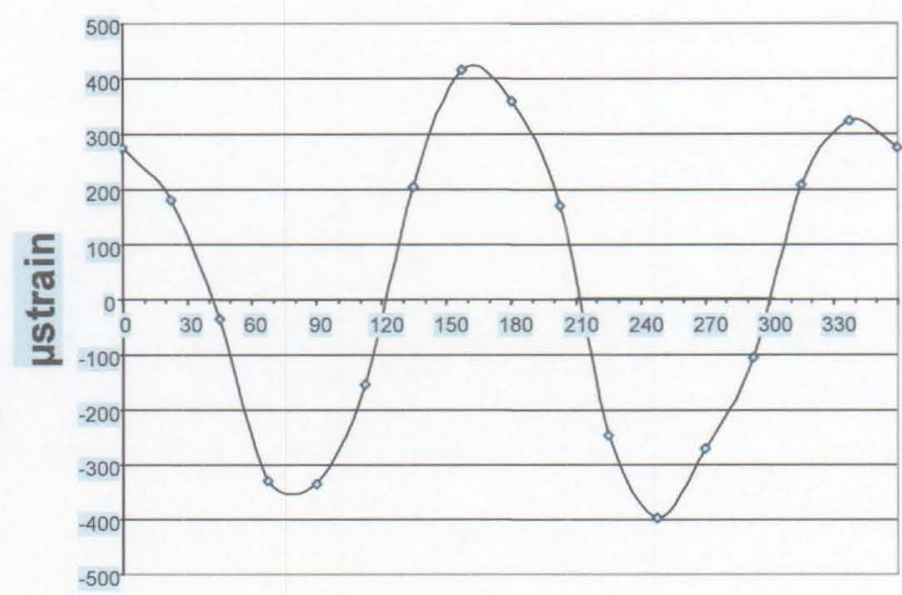

Circumferential location (deg) 
- $1^{\text {st: }}$ thick, rigid wall to provide baseline of separation characteristics.

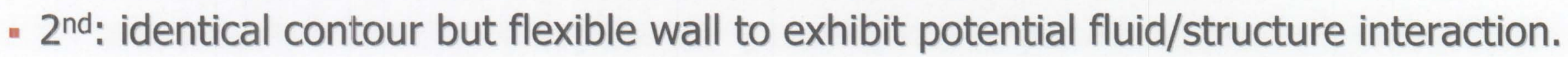

Structural Dynamic Analysis

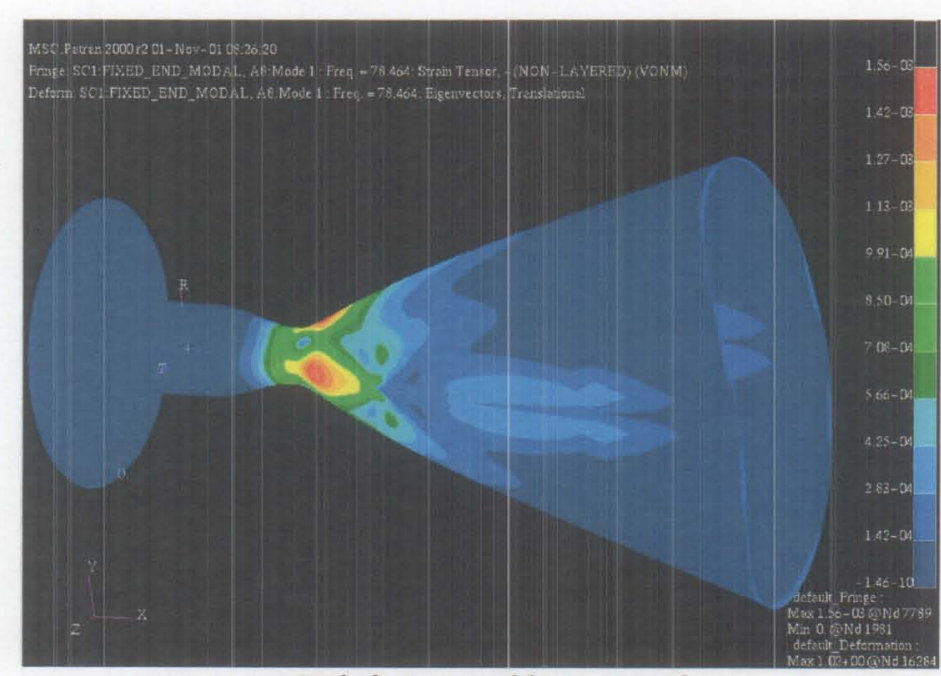

Thin wall nozzle

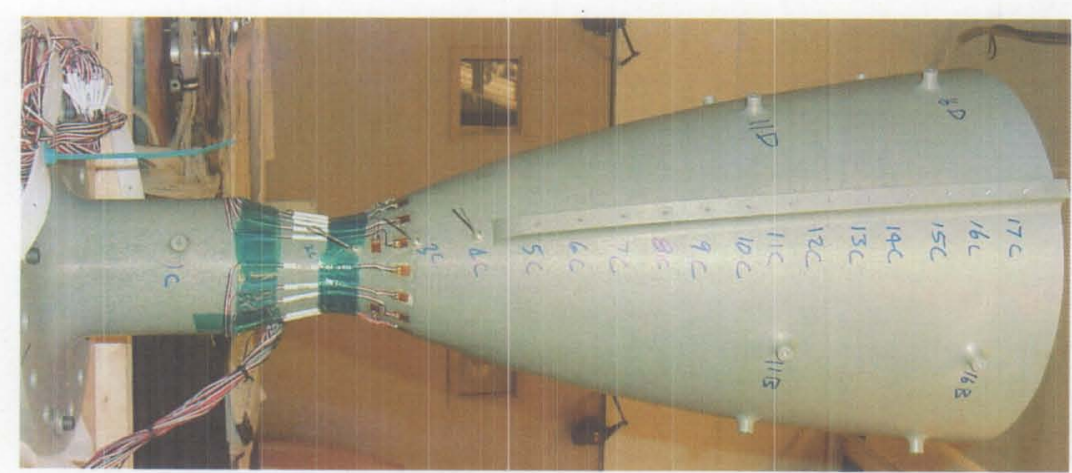

Thick wall nozzle in NTF

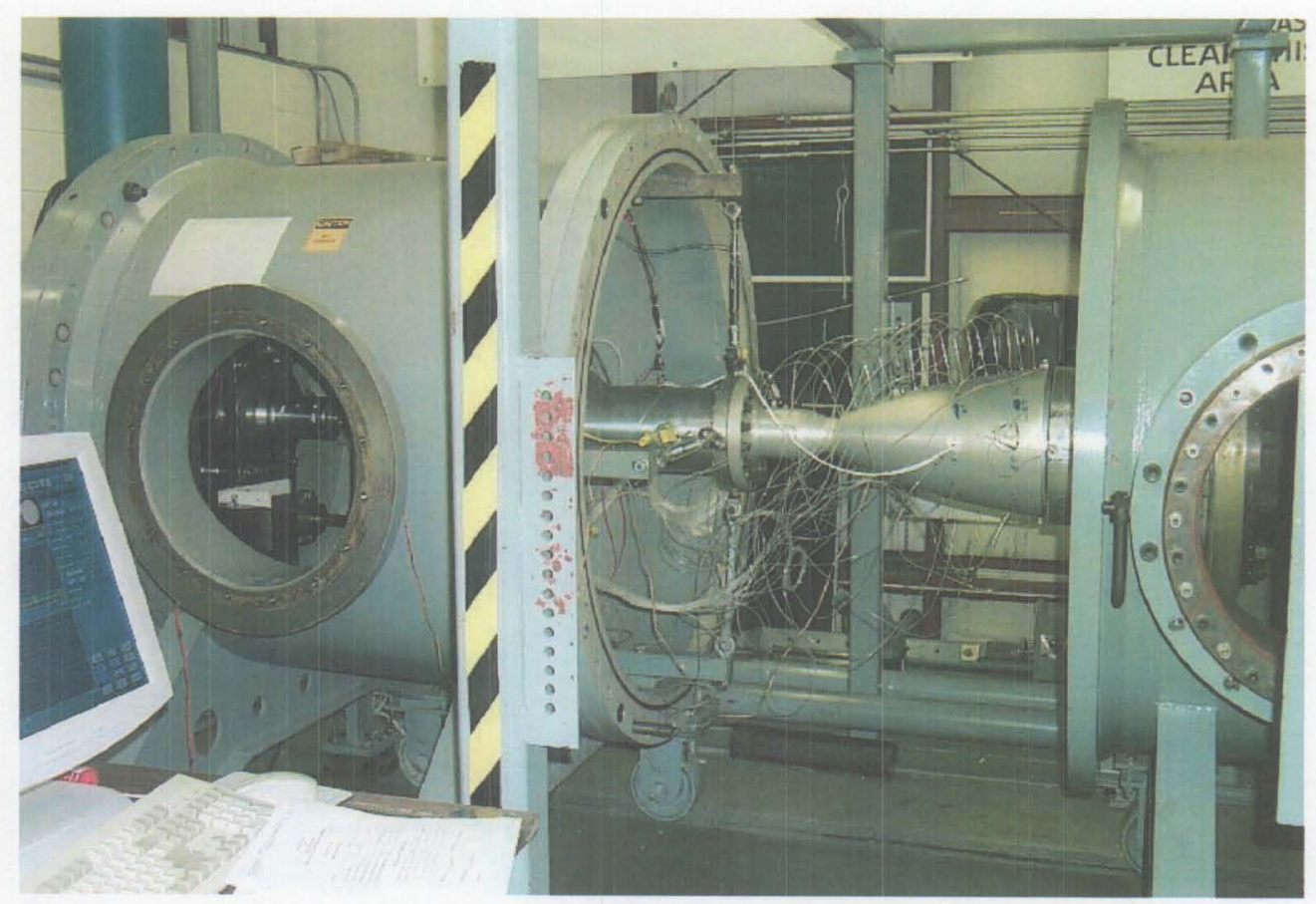




\section{Test Data Analysis}

- Strain Gage data was combed for all thin wall nozzle runs to investigate nozzle response characteristics in high amplitude regimes.

- Once high amplitude regimes were identified, Strain Spectral Densities, Time Histories, Frequency Tracking, Frequency Amplitude tracking and shape decompositions were used to characterize response.

- In every instance of a high strain regime, the nozzle 2ND mode was the dominant response.
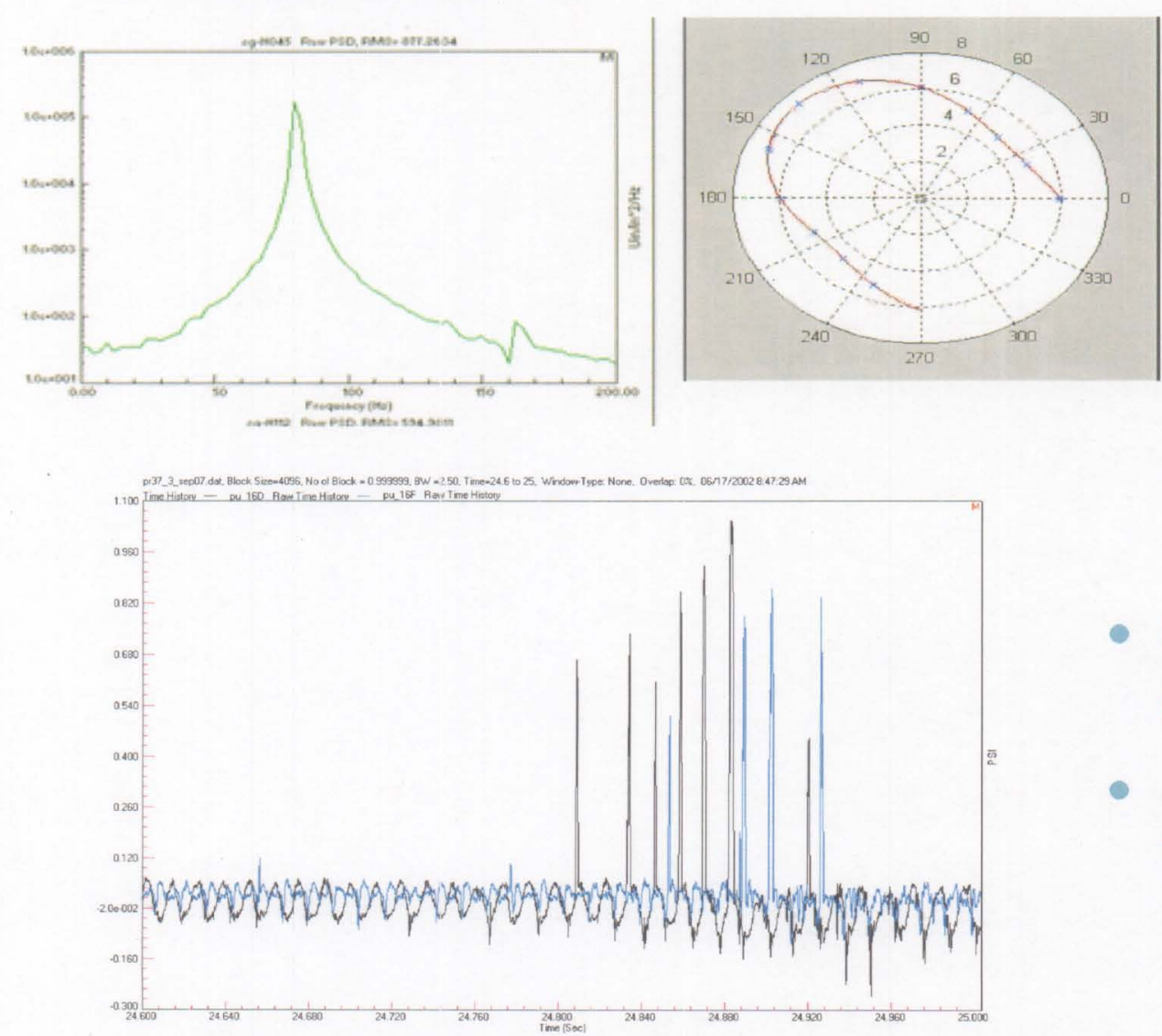

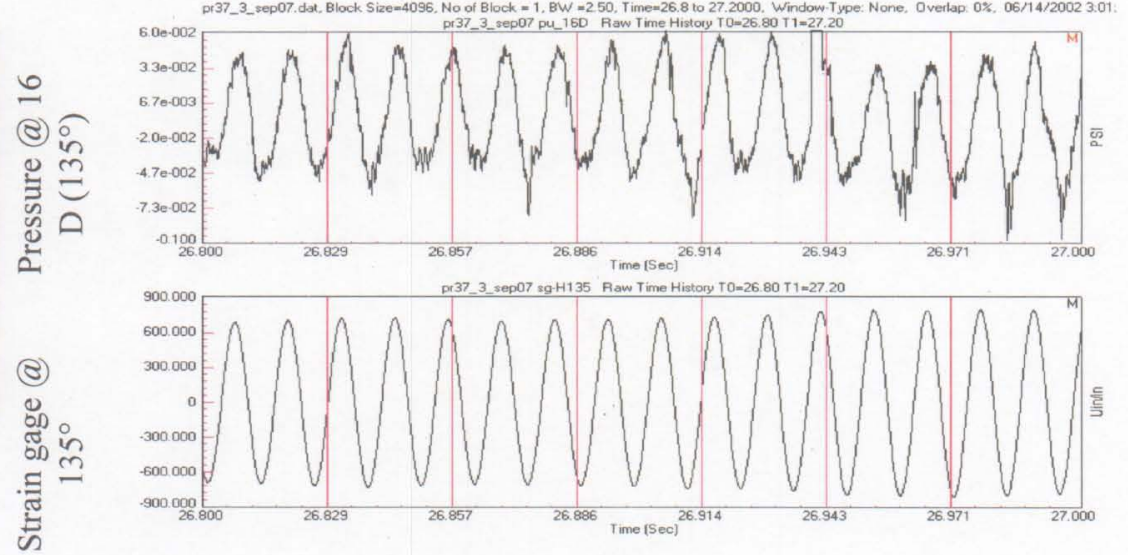

- Separation spikes also at same frequency as $2^{\mathrm{ND}}$

- Fluid/structure self-excited vibration loop verified. 


\section{Simplified Fluid/Structure Analysis Methodology}

- To take next step of being able to predict nozzle response, fully accurate methodology would need full 3D CFD coupled with accurate structural dynamic model using feedback control.

- A simplified model was formulated instead that would tie a 2D CFD calculation of the internal pressure field of a nozzle undergoing structurally defined flow separation with nozzle structural dynamic response at a single instance in time.

- The steps to this formulating this model are as follows: 


\section{Estimation of Deformation Field}

- Assume nozzle is responding in 2 Nodal Diameter self-excited bounded feedback loop and has reached maximum response.

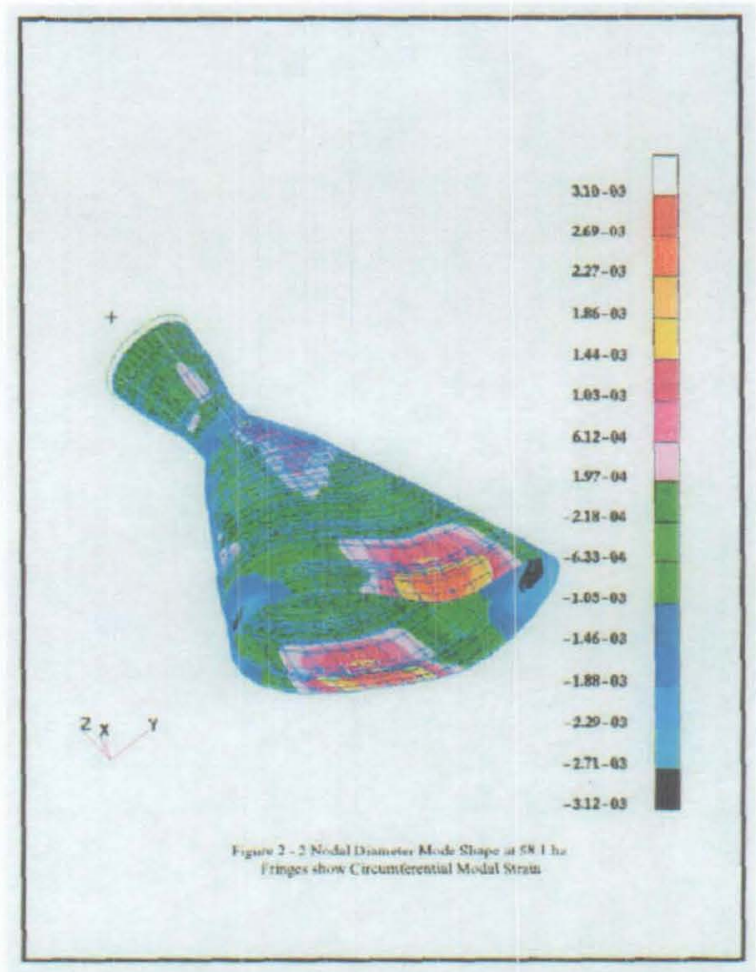

- Take highest strain gage responses from the cold flow testing and compare to normalized strains from finite element modal analysis of the nozzle.

- Comparison yields a strain scale factor.

- Normalized deflection from modal analysis then multiplied by scale factor to obtain deformations of nozzle.

- Nozzle deformed contours were created every 22.5 degrees from 0 to 90 .

- Resulting deformations much higher than video evidence, so $20 \%$ of this deformation used as starting point for fluid dynamics calculations. 


\section{Generation of Pressure Profiles}

- 2D Kinetics (TDK) CFD methodology used to generate nozzle internal flow fields for each of the deformed profiles.

- Wall pressure vs. axial location curves generated.

- Curves manually altered to show nozzle separation at locations where wall pressure is 0.29 of the ambient pressure for each contour.

- Pressure downstream of each contour's separation location then assumed to rise to 0.98 of ambient pressure. 


\section{Pressure Profiles}

Wall Pressure and Delta Pressure, Seperated
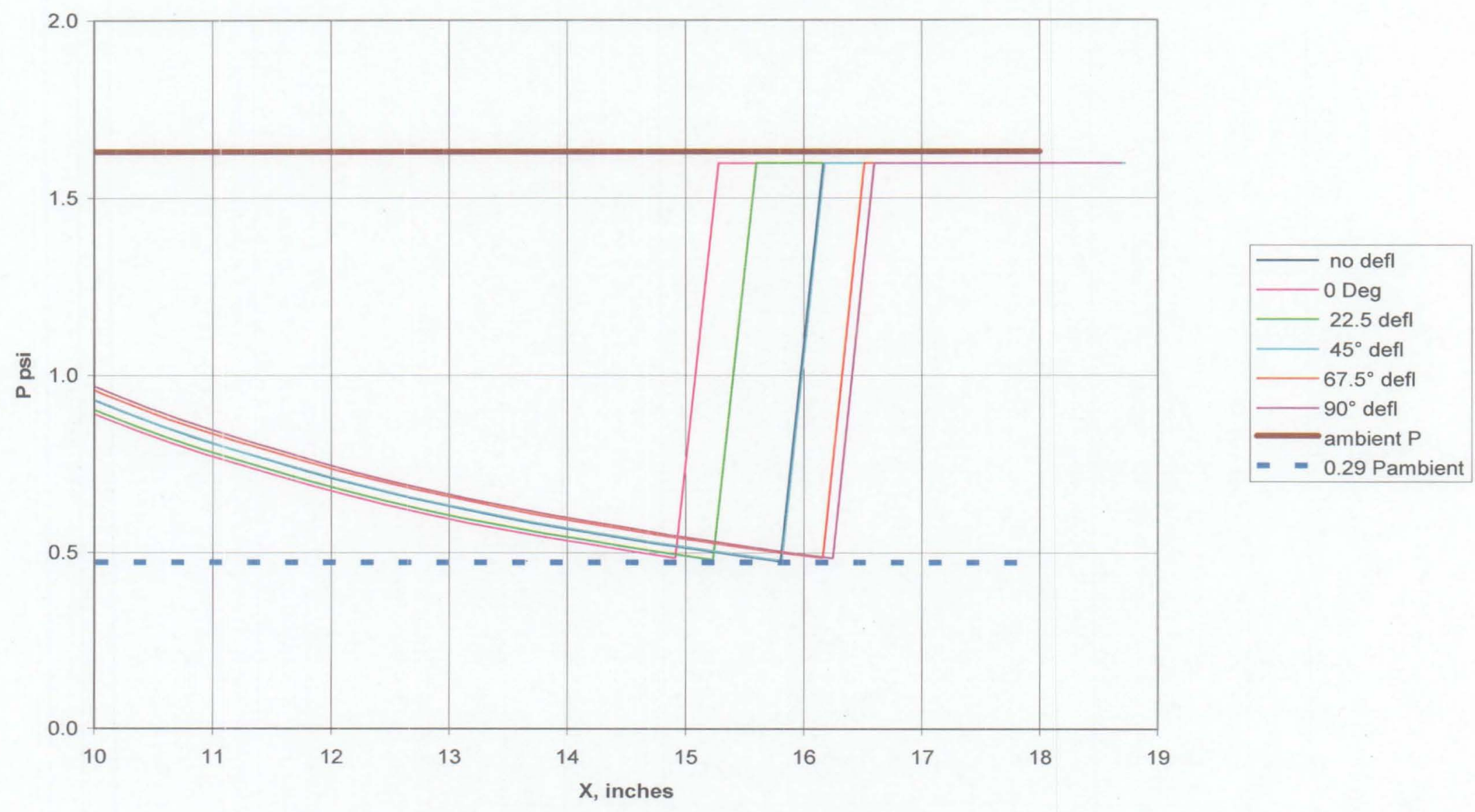


\section{Add time variation to pressure field}

- Subtract out ambient pressure.

- Interpolate curves around 360 degrees of nozzle.

- Fluctuate curves at natural frequency of nozzle about mean $\Delta p$ at 6 axial positions.

- Square wave at second to last excitation plane to account for moving separation plane.

- Export time histories for 16 circumferential positions for 6 axial planes into NASTRAN.

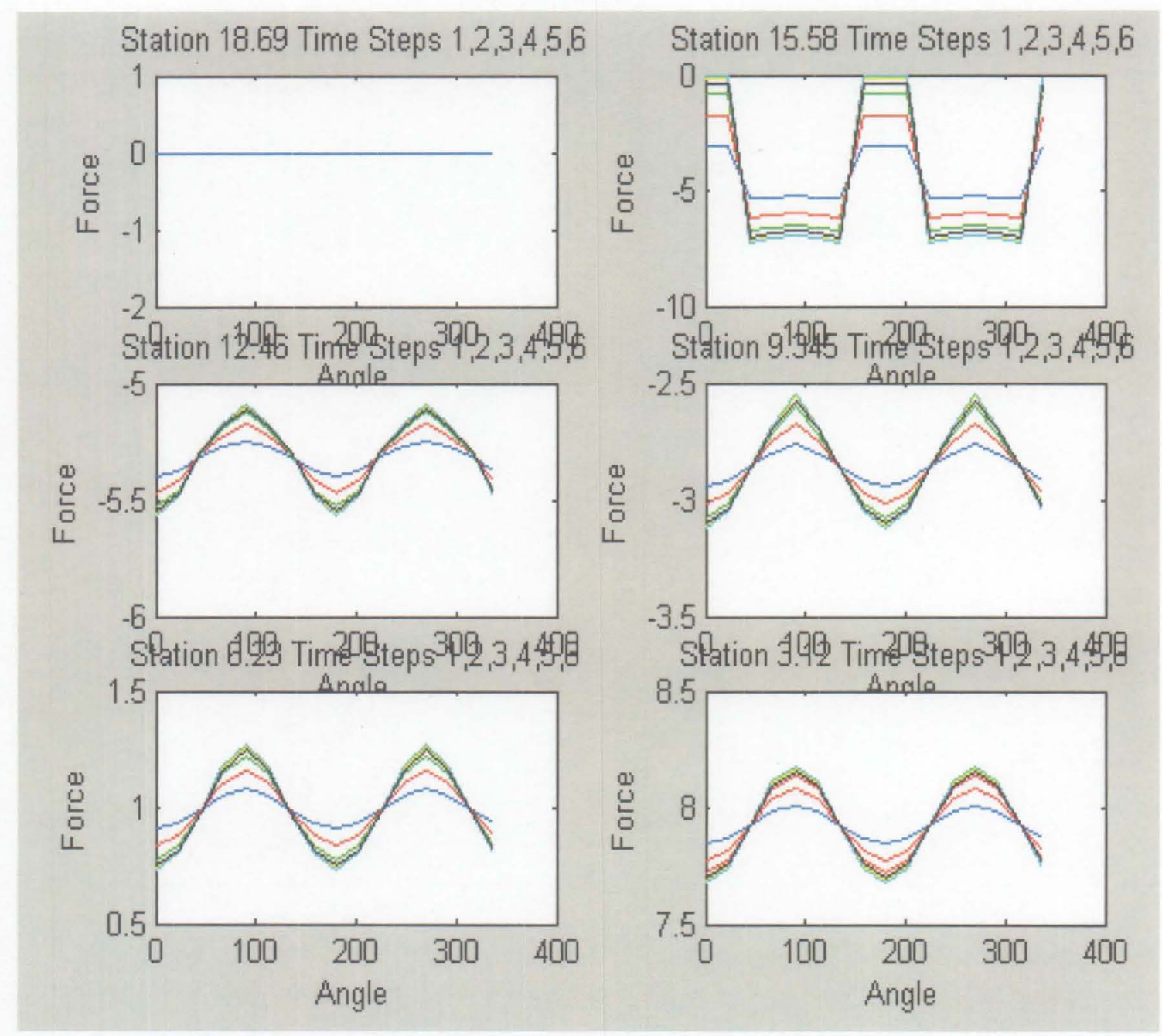


- Transient forced response analysis performed on nozzle model by applying forces to nodes on nozzle.

- Both modal test derived damping of the nozzle and experimentally calculated cold flow test systems damping used in analysis.

- 256 time steps chosen to allow for easy Fast Fourier Transform and Power Spectral Density creation from resulting strains.

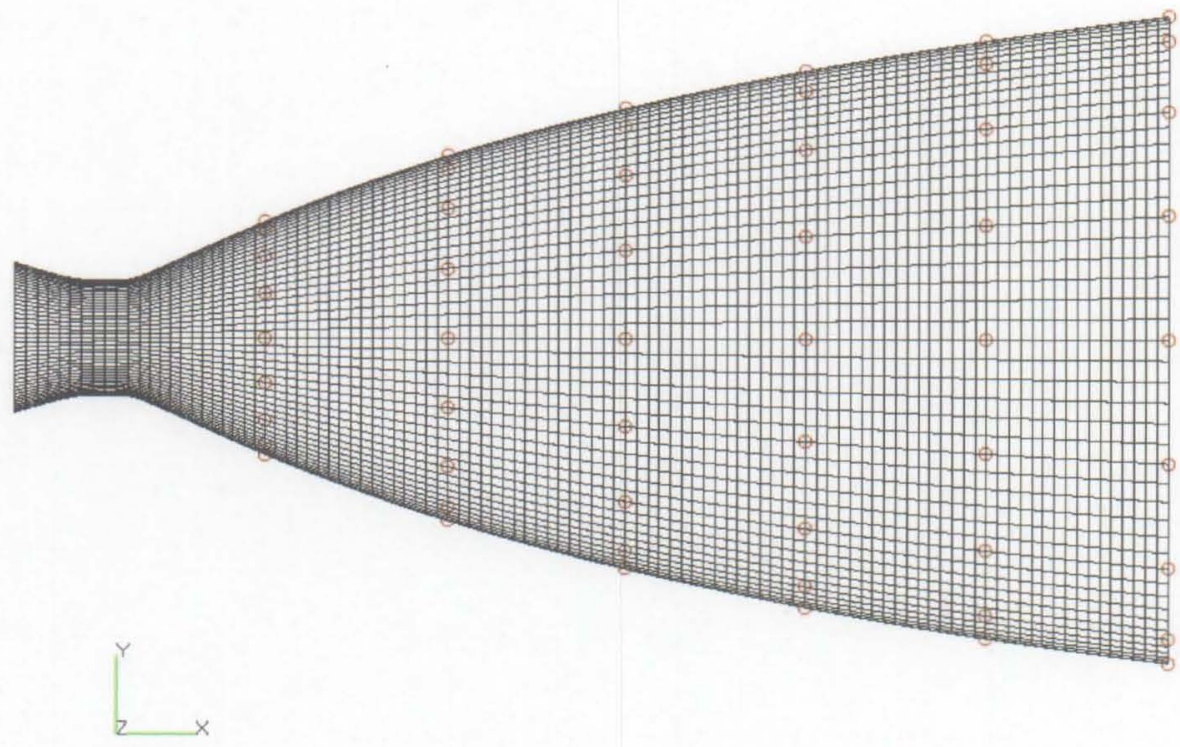




\section{Temporal and Frequency Results}

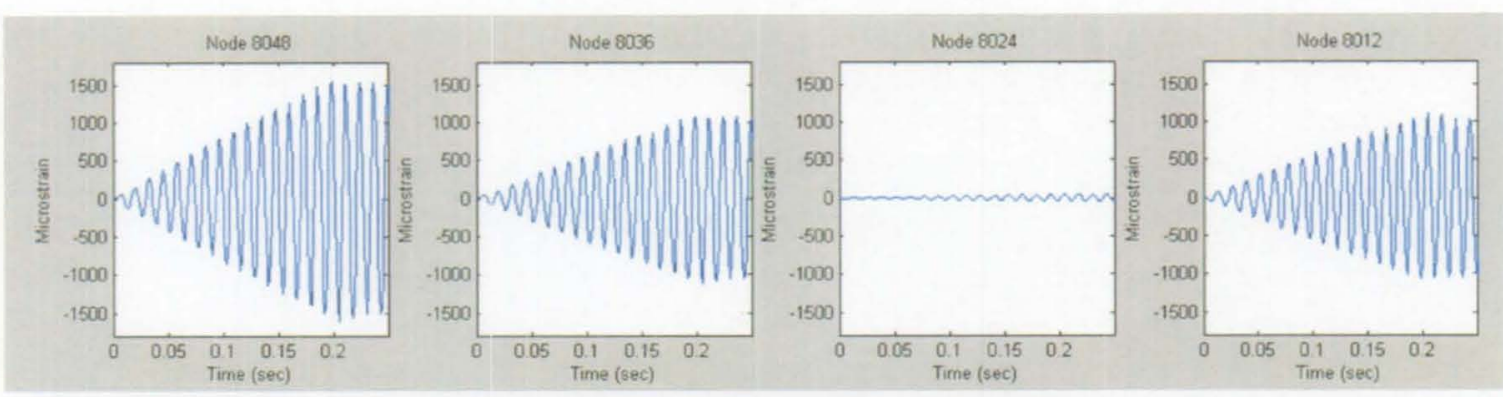

Figure 9. Analytical Circumferential Strain Time History Results
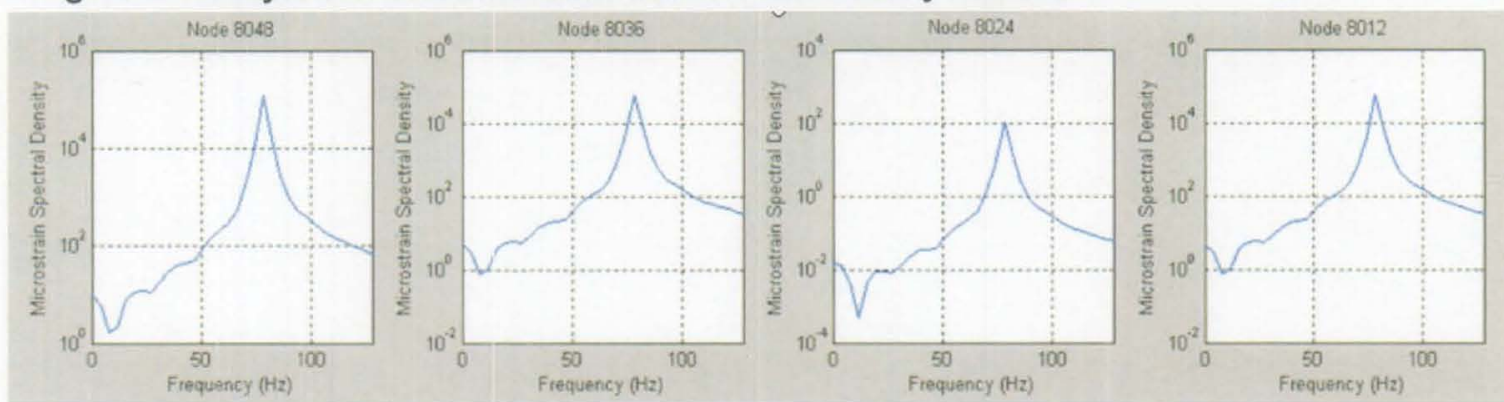

Figure 10. Analytical Circumferential Strain PSD Results
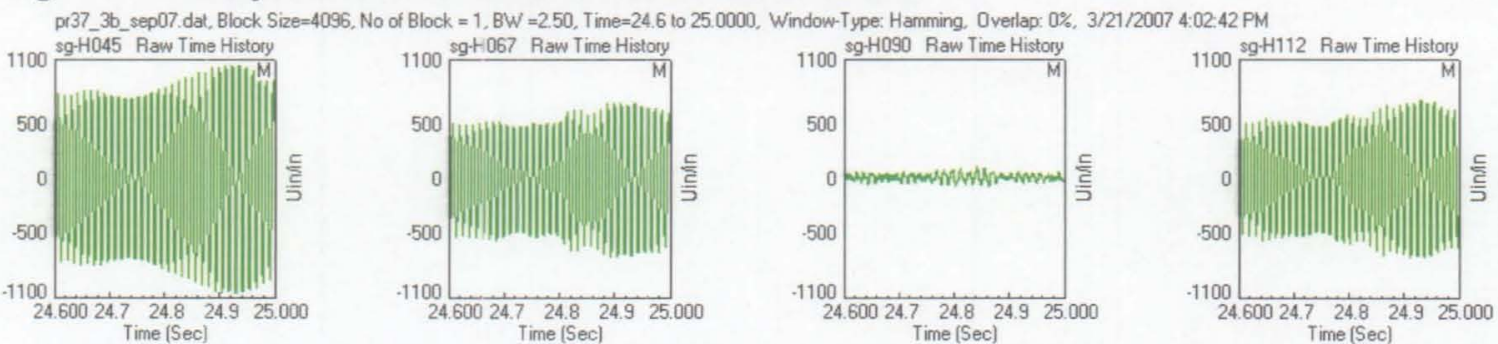

Figure 11. Experimental Circumferential Strain Time History Results
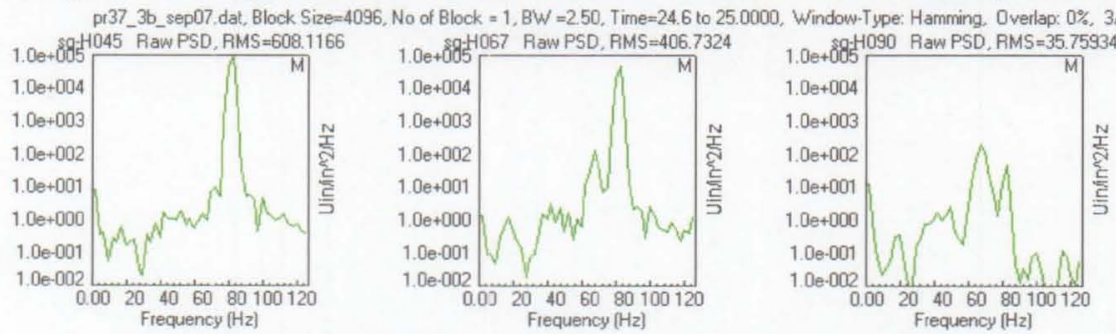

Figure 12. Experimental Circumferential Strain PSD Results
- Analysis Hoop Strain magnitude is $40 \%$ higher than that of test.

- Recent examination of results have shown steady-state not quite reached.

- Frequency content compares well.

- Axial strain maximum magnitude difficult to compare due to limited strain gages in high strain gradient regime. 


\section{Spatial Results}
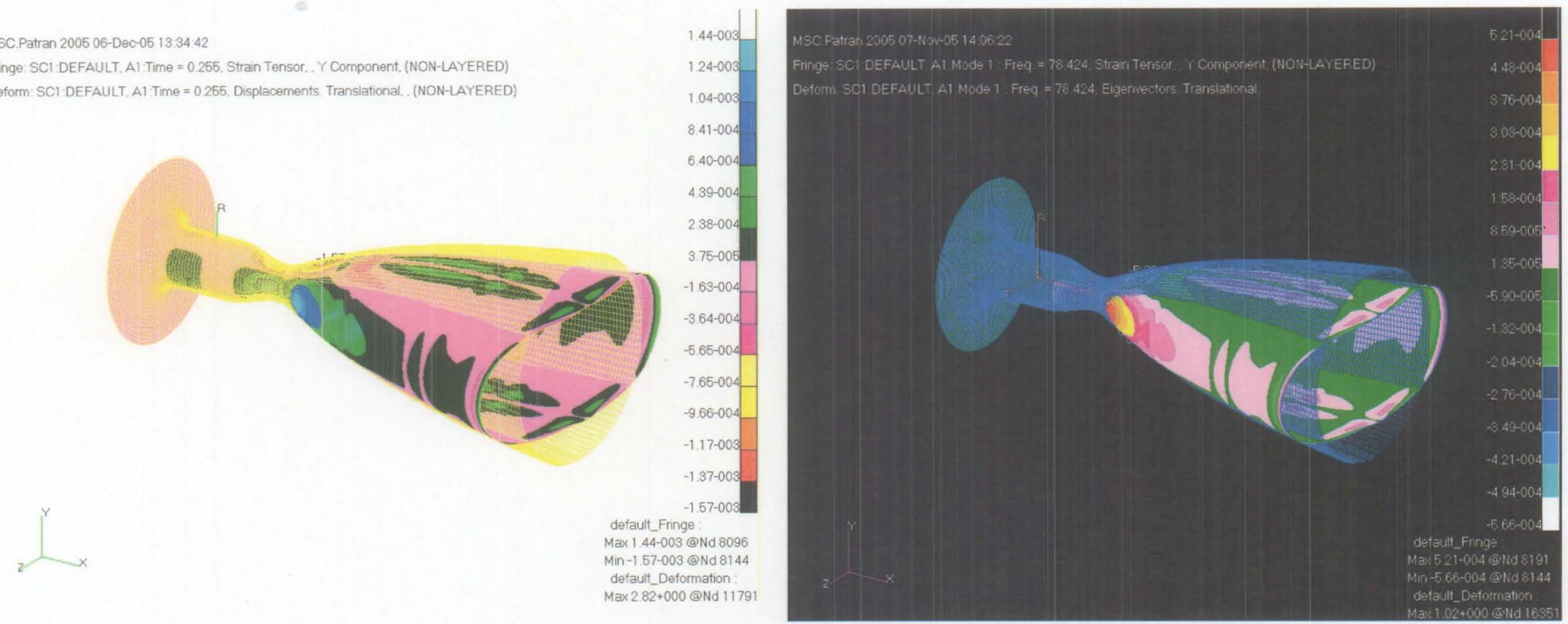

- Analysis strain field takes the exact form of nozzle two modal diameter shape. 


\section{Sources of Error}

- Magnitude of originally calculated deformation field

- Higher estimate would yield even larger overprediction.

- Damping estimation; modal test showed $0.22 \%$, halfpower gave $0.8 \%$.

- Both cases run, even $0.8 \%$ yielded $60 \%$ overprediction.

- Location of separation - data have shown value can range between 0.25 and 0.4 .

- If higher value used, separation loads would be lower, analysis would yield lower, closer results. 


\section{Conclusions}

- Initial formulation of analysis model that approximates temporal and spatial fluid force field interacting with structural dynamic model of rocket nozzle undergoing steady-state flow separation.

- Results reinforce previous data indicating that this interaction generates a self-excited vibration loop.

- Initial analytical response results over-predict measured strain response of the nozzle.

- Future work on this project includes resolution of errors identified during this initial study.

- 3D fluid effects could be captured in this analysis by performed a 90 degree section CFD analysis of the nozzle in the deformed state.

- Validated analytical model would be used to predict nozzle side load forcing functions for rocket engine design. 